
ImmunoTherapy of Cancer

\section{An engineered oncolytic vaccinia virus encoding a single-chain variable fragment against TIGIT induces effective antitumor immunity and synergizes with PD-1 or LAG-3 blockade}

To cite: Zuo S, Wei M, Xu T, et al. An engineered oncolytic vaccinia virus encoding a single-chain variable fragment against TIGIT induces effective antitumor immunity and synergizes with PD-1 or LAG-3 blockade. Journal for ImmunoTherapy of Cancer 2021;9:e002843. doi:10.1136/ jitc-2021-002843

- Additional supplemental material is published online only. To view, please visit the journal online (http://dx.doi.org/10. 1136/jitc-2021-002843).

SZ, MW and TX contributed equally.

Accepted 23 November 2021

Check for updates

(C) Author(s) (or their employer(s)) 2021. Re-use permitted under CC BY-NC. No commercial re-use. See rights and permissions. Published by BMJ.

${ }^{1}$ Jiangsu Key Laboratory of Molecular Medicine, Medical School of Nanjing University, Nanjing, Jiangsu, China ${ }^{2}$ Laboratory for Translational Medicine, Chifeng Municipal Hospital, Chifeng Clinical Medical School, Inner Mongolia Medical University, Chifeng, Inner Mongolia, China

Correspondence to Professor Jiwu Wei; wjw@nju.edu.cn

Dr Jie Dong; dongjie@nju.edu.cn

\section{ABSTRACT}

Background In addition to directly lysing tumors, oncolytic viruses also induce antitumor immunity by recruiting and activating immune cells in the local tumor microenvironment. However, the activation of the immune cells induced by oncolytic viruses is always accompanied by high-level expression of immune checkpoints in these cells, which may reduce the efficacy of the oncolytic viruses. The aim of this study is to arm the oncolytic vaccinia virus (VV) with immune checkpoint blockade to enhance its antitumor efficacy.

Methods Through homologous recombination with the parental W, an engineered W-scFv-TIGIT was produced, which encodes a single-chain variable fragment (scFv) targeting T-cell immunoglobulin and ITIM domain (TIGIT). The antitumor efficacy of the W-scFv-TIGIT was explored in several subcutaneous and ascites tumor models. The antitumor efficacy of V-scFv-TIGIT combined with programmed cell death 1 (PD-1) or lymphocyte-activation gene 3 (LAG-3) blockade was also investigated.

Results The VV-scFv-TIGIT effectively replicated in tumor cells and lysed them, and prompt the infected tumor cells to secret the functional scFv-TIGIT. Compared with control VV, intratumoral injection of VV-scFv-TIGIT in several mouse subcutaneous tumor models showed superior antitumor efficacy, accompanied by more T cell infiltration and a higher degree of $\mathrm{CD}^{+} \mathrm{T}$ cells activation. Intraperitoneal injection of V-scFv-TIGIT in a mouse model of malignant ascites also significantly improved $\mathrm{T}$ cell infiltration and $\mathrm{CD} 8^{+} \mathrm{T}$ cell activation, resulting in more than $90 \%$ of the tumor-bearing mice being cured. Furthermore, the antitumor immune response induced by VV-scFv-TIGIT was dependent on $\mathrm{CD}^{+} \mathrm{T}$ cells which mediated a long-term immunological memory and a systemic antitumor immunity against the same tumor. Finally, the additional combination of PD-1 or LAG-3 blockade further enhanced the antitumor efficacy of VV-scFv-TIGIT, increasing the complete response rate of tumor-bearing mice.

Conclusions Oncolytic virotherapy using engineered VV-scFv-TIGIT was an effective strategy for cancer immunotherapy. Administration of V-scFv-TIGIT caused a profound reshaping of the suppressive tumor microenvironment from 'cold' to 'hot' status. VV-scFv-TIGIT also synergized with PD-1 or LAG-3 blockade to achieve a complete response to tumors with poor response to $\mathrm{V}$ or immune checkpoint blockade monotherapy.

\section{BACKGROUND}

Oncolytic viruses (OVs) are natural or genetically engineered viruses that can selectively replicate in tumor cells and kill them without the destruction of normal cells. ${ }^{1}$ Currently, herpes simplex virus (HSV), adenovirus (ADV), and vaccinia virus (VV or VACV) are the three most commonly engineered viruses used in preclinical studies or clinical trials for cancer treatment. ${ }^{2}$ As a vaccine used to eradicate smallpox, VV has become the most widely studied virus in the poxviridae family. Several inherent biological characteristics of $\mathrm{VV}$ enable it to show advantages as an OV. First, VV has a large genome (approximately $192 \mathrm{kbp}$ ), which allows it to accommodate the insertion of large foreign DNA fragments into the viral genome. ${ }^{3}$ Second, VV does not enter the nucleus but completes the entire cycle of replication in the cytoplasm, which provides a safety advantage for $\mathrm{VV}$ as an $\mathrm{OV}$ since the viral DNA has less probability to integrate into the host genome. ${ }^{4}$ Third, the worldwide smallpox vaccination has accumulated a lot of safety data for the clinical application of oncolytic VV. ${ }^{3}$ Finally, VV can recruit immune cells and stimulate the body to produce a systemic antitumor immune response, which is essential for removing distant and metastatic tumor cells. ${ }^{5}$ These characteristics make $\mathrm{VV}$ one of the most promising candidates for oncolytic virotherapy. Pexastimogene devacirepvec (Pexa-Vec, JX-594) is an engineered $\mathrm{VV}$ in which a gene encoding granulocytemacrophage colony-stimulating factor (GM$\mathrm{CSF})$ is inserted as an immune adjuvant. ${ }^{6}$ 
Pexa-Vec is currently being evaluated in phase III clinical trials in patients with advanced hepatocellular carcinoma. However, it is still uncertain whether immune activation caused by genetic modification will produce better therapeutic effects.

Although the initial studies mainly focused on how to improve the selectivity and toxicity of OVs to tumor cells, oncolytic virotherapy is now regarded as an immunotherapy method for cancer due to its ability to increase tumor-specific effector and memory $\mathrm{T}$ cells. ${ }^{78}$ These activated $\mathrm{T}$ cells can strengthen the oncolysis of the virus at the site of virus injection, and they can migrate to a certain distance to kill the metastasized tumor cells, thereby producing an abscopal effect. ${ }^{9}$ Since the Food and Drug Administration (FDA)-approved talimogene laherparepvec (T-VEC, a GM-CSF-modified HSV-1) for the treatment of metastatic melanoma in $2015,{ }^{10}$ oncolytic virotherapy has received more attention in the field of cancer immunotherapy. However, the efficacy of OVs against solid tumors has not yet reached expectations, mainly due to the limitations of many factors such as physical barriers, tumor heterogeneity, and immunosuppressive tumor microenvironment (TME). ${ }^{11}$ There are multiple immunosuppressive pathways involved in the TME. Immune checkpoints (such as cytotoxic T-lymphocyte antigen-4 (CTLA-4), programmed cell death 1 (PD1), T-cell immunoglobulin and ITIM domain (TIGIT), T-cell immunoglobulin and mucin domain-3 (TIM-3), and lymphocyte-activation gene 3 (LAG-3)) are upregulated in tumor-infiltrating lymphocytes (TILs), which contribute to the formation of an immunosuppressive TME. $^{12}$

The combination of OVs and immune checkpoint blockade (ICB) was explored to overcome the immunosuppressive TME. ${ }^{13}$ Recently, a phase Ib/II study demonstrated that the combined use of T-VEC and ipilimumab (Yervoy, a CTLA-4 blockade) appeared to be more effective than T-VEC or ipilimumab monotherapy. ${ }^{14}$ Another phase Ib clinical study showed that T-VEC synergized with pembrolizumab (Keytruda, the first FDA-approved PD-1 blockade) and improved the efficacy of pembrolizumab in the treatment of melanoma. ${ }^{15}$ In addition, OVs can be equipped with immune activators (such as cytokines, ${ }^{16} 17$ chemokines, ${ }^{18}$ costimulatory molecules, ${ }^{11}$ and immune checkpoint antibodies ${ }^{19-24}$ ) through genetic modification to further enhance the recruitment of T cells in the TME and reshape the immunosuppressive TME. ${ }^{25}$

Beyond CTLA-4 and PD-1, TIGIT is one of the most concerned immune checkpoints as the target of cancer immunotherapy. ${ }^{26}$ TIGIT belongs to an emerging member of the immunoglobulin superfamily, which also includes poliovirus receptor (PVR)-related immunoglobulin domain-containing (PVRIG, CD112R), T cell-activated increased late expression protein (Tactile, CD96), and DNAX accessory molecule-1 (DNAM-1, CD226). ${ }^{27}$ TIGIT, PVRIG, and Tactile are co-inhibitory receptors whereas DNAM-1 is a co-stimulatory receptor. These receptors mainly interact with the nectin and nectin-like (necl) family ligands (including PVR (also known as Necl-5, CD155) and PVR-related 1-4 (PVRL1-4, Nectin-1-4, CD111-114)) to form a complex regulatory network to control the activation of natural killer (NK) cells and T cells. ${ }^{28}$ TIGIT is weakly expressed on naive $\mathrm{T}$ cells but frequently overexpressed on activated NK, $\mathrm{CD}^{+} \mathrm{T}$ cells, and regulatory $\mathrm{T}$ cells (Tregs) ${ }^{29}$ Furthermore, TIGIT is co-expressed with other co-inhibitory receptors (such as PD-1, CTLA-4, TIM-3, and LAG-3) on TILs, which is related to the exhaustion of activated $\mathrm{CD} 8^{+}$ T cells. ${ }^{30}$ For these reasons, TIGIT has been considered a lucrative cancer immunotherapy candidate and several TIGIT monoclonal antibodies have been developed both in preclinical studies and clinical trials, particularly in combination with PD-1 or the other ICBs. ${ }^{31}{ }^{32}$ Recently, studies have shown that TIGIT blockade significantly improved the antitumor efficacy of an oncolytic HSV-1 encoding a single-chain variable fragment $(\mathrm{scFv})$ against PD- $1 .{ }^{33}$ However, there are no reports on the use of scFv against TIGIT to arm the OV.

In the present study, we generated an engineered oncolytic VV, VV-scFv-TIGIT, which encoded a TIGIT scFv, and systematically investigated its antitumor efficacy in several mouse subcutaneous-tumor models and an ascites tumor model. We also investigated the efficacy of the combined application of the VV-scFv-TIGIT with PD-1 or LAG-3 blockade on colon tumor models.

\section{MATERIALS AND METHODS Cell lines}

H22 cells were obtained from the China Center for Type Culture Collection (ID: 3111C0001CCC000309, Wuhan, China). HEK293 (Cat\# CRL-1573), Hela-S3 (Cat\# CCL2.2), 4T1 (Cat\# CRL-2539), B16/F10 (Cat\# CRL-6475), and CT26 (Cat\# CRL-2638) cell lines were purchased from the American Type Culture Collection (ATCC; Manassas, USA). MC38 cells (RRID: CVCL_B288) were obtained from the National Cancer Institute (USA). HEK293, Hela-S3, 4T1, MC38, CT26, and B16/F10 cells were maintained in Dulbecco's modified Eagle's medium (DMEM; Cat\# 11965092, Gibco-Thermo Fisher Scientific, USA) supplemented with $10 \%$ fetal bovine serum (FBS; Cat\#16000044, Gibco). H22 cells were maintained in Roswell Park Memorial Institute 1640 medium (Cat\# 11875093, Gibco) supplemented with 10\% FBS. Under suspension conditions, Hela-S3 cells were maintained in a spinner flask (Jetbiofil, Guangzhou, China) in a serumfree medium (Cat\# H740KJ, Basalmedia, Shanghai, China). All cells were cultured in an incubator at $37^{\circ} \mathrm{C}$ and $5 \% \mathrm{CO}_{2}$ atmosphere.

\section{Construction of recombinant oncolytic VV}

The sequences of the variable domain of the heavy chain $(\mathrm{VH})$ and the variable domain of the light chain (VL) are based on the hamster antimouse TIGIT monoclonal antibody (Clone 10a7) in the patent US20090258013A1. The gene fragment encoding scFv against TIGIT (scFv-TIGIT) 
consisted of a human interleukin-2 (IL-2) signal peptide, an HA-tag, a VH, a (GGGGS) $\times 3$ linker, and a VL. This gene fragment was synthesized by GenScript (Nanjing, China) and subcloned into a shuttle plasmid pVV-Control (online supplemental figure S1A) to construct the recombinant plasmid pVV-scFv-TIGIT (online supplemental figure $\mathrm{S} 1 \mathrm{~B}$ ). In this plasmid, the expression of the $\mathrm{scFv}$ TIGIT is driven by a synthesized early/later promoter $(\mathrm{pSE} / \mathrm{L})$, while the expression of the enhanced green fluorescent protein (EGFP; reporter gene) and the guanine-hypoxanthine phosphoribosyl transferase (GPT; screening gene) is driven by a $7.5 \mathrm{~K}$ early/later promoter of VV.

To generate VV-scFv-TIGIT, the shuttle plasmid pVVscFv-TIGIT was used for homologous recombination with a western reserve strain of VV (WR-VV; Cat\# VR-1354; ATCC). Briefly, HEK293 cells were infected with the WR-VV at a multiplicity of infection (MOI) of 1 for 2 hours and then transfected with the pVV-scFv-TIGIT using the jetPRIME transfection reagent (Cat\# 114-15, Polyplustransfection; Illkirch, France). Forty-eight hours later, the formed EGFP-positive plaques were picked and seeded in plates with Hela-S3 cells. The conditional DMEM medium containing $250 \mu \mathrm{g} / \mathrm{mL}$ xanthine (Cat\# A601197, Sangon Biotech, Shanghai, China), $25 \mu \mathrm{g} / \mathrm{mL}$ mycophenolic acid (Cat\# A600640, Sangon), and $15 \mu \mathrm{g} / \mathrm{mL}$ hypoxanthine (Cat\# A500336, Sangon) was used to inhibit the growth of WR-VV. After several cycles of picking and seeding, both PCR and DNA sequencing were used to confirm that the recombinant virus was no longer adulterated with WR-VV. Then the purified virus was gradually expanded by Hela-S3 cells in 6-well plates, cell culture dishes, and cell culture spinner flasks. Similarly, pVV-Control was used for homologous recombination with WR-VV to generate a control VV, namely VV-Control. The virus titer was determined by a TCID50 method. The calculation formula is as follows: virus titer $=0.7 \times 10 \times 10^{(1+\mathrm{S}(\mathrm{D}-0.5))}$, where $\mathrm{S}$ is $\log 10$ (dilution), D is the sum of the positive ratios of EGFP in each dilution.

\section{Western blot analysis}

Tumor cells were seeded in 6-well plates at a density of $5 \times 10^{5}$ cells per well and infected with VV-scFv-TIGIT or VV-Control at MOI of 1 . After a 48-hour incubation, the cell culture supernatants were collected, and $10 \mu \mathrm{L}$ of the supernatants was taken out and mixed with a $2 \times$ loading buffer (Cat\# P0015, Beyotime, Shanghai, China) in equal proportions. The protein samples were heated at $100^{\circ} \mathrm{C}$ for $5 \mathrm{~min}$ and then loaded on a sodium dodecyl-sulfate polyacrylamide gel electrophoresis (SDS-PAGE) gel for electrophoresis. After the electrophoresis, the protein on the PAGE gel was transferred to a polyvinylidene fluoride (PVDF) membrane (Cat\# K5MA6539B, Merck Millipore, Germany) using a semi-dry membrane transfer instrument. Then the PVDF membrane was incubated with a mouse anti-HA tag antibody (Clone 5E11D8, Cat\# A01244, GenScript, Nanjing, China) at $4^{\circ} \mathrm{C}$ overnight, and then incubated with horseradish peroxidase
(HRP)-labeled goat antimouse IgG $(\mathrm{H}+\mathrm{L})$ (Cat\# A0216, Beyotime) at room temperature (RT) for 2 hours. After the incubation, an enhanced chemiluminescent kit (Cat\# FD8000, FDbio, Hangzhou, China) was used to visualize the protein bands. A purified $\sim 40 \mathrm{kDa}$ Multiple Tag fusion protein (Cat\# M0101, GenScript, Nanjing, China) was serve as a positive control for western blot analyses.

\section{TIGIT binding and competition assay}

For binding assay, 96-well plates were coated with recombinant mouse TIGIT protein (r-TIGIT; Cat\# 50939-M38H, Sino Biological, Beijing, China) at a concentration of $10 \mu \mathrm{g} / \mathrm{mL}$. Then, supernatants containing scFv-TIGIT (prepared by infection of Hela-S3 cells with $\mathrm{VV}-\mathrm{scF}$ TIGIT) were collected and added to the precoated wells and incubated at $4^{\circ} \mathrm{C}$ for 12 hours. After 3-time of wash, the mouse anti-HA-tag antibody $(0.1 \mu \mathrm{g} / \mathrm{mL})$ was added to each well and incubated at RT for 1 hour. Diluted (1: 2000) HRP-labeled secondary antibody (Cat\# A0216, Beyotime) was added to each well after 3-time of wash and incubated at RT for 1 hour. After 3-time of wash, $100 \mu \mathrm{L}$ TMB substrate was added to each well and incubated at RT for $20 \mathrm{~min}$. The optical density (OD) value was measured at $450 \mathrm{~nm}$ using an absorbance microplate reader after $100 \mu \mathrm{L}$ of $2 \mathrm{~N}$ sulfuric acid was added to stop the reaction.

For the competition assay, the coating procedure of the 96-well plate and the addition of the supernatants containing scFv-TIGIT were similar to the binding assay. After 3-time of wash, the cell culture supernatants containing Lucia-fused PVR (Lucia-PVR; prepared by transfection of HEK293 cells with a pVV-EF1 $\alpha$-LuciamPVR plasmid; online supplemental figure S1C) were added to the wells and incubated at $4^{\circ} \mathrm{C}$ for 12 hours. After 5-time of wash, $50 \mu \mathrm{L}$ of QUANTI-Luc substrate (Cat\# rep-qlc1, InvivoGen, USA) was added to the wells, and the luciferase activity was immediately detected by the Glomax96 microplate luminescence detector.

\section{Crystal violet staining}

B16/F10, 4T1, MC38, and CT26 cells were seeded in 96-well plates at a density of $5 \times 10^{3}$ cells per well, respectively, and cultured in an incubator at $37^{\circ} \mathrm{C}$ with $5 \% \mathrm{CO}_{2}$ atmosphere. When the tumor cells reached $90 \%$ confluence, VVs were added to the wells at MOI of $0,0.1,1$, 5 , and 10 . After a 72 hours incubation, the supernatants were removed and the $0.2 \%(\mathrm{w} / \mathrm{v})$ crystal violet solution (Cat\# C0121, Beyotime) was added to the wells for staining. After a 5 min incubation, the staining solution was removed from the wells and rinsed with $\mathrm{dd} \mathrm{H}_{2} \mathrm{O} 5$ times. The image was acquired using a scanner. ImageJ software (V.1.53) was used to calculate the area of living cells. The percentage was calculated using the area of each well relative to the area of the well with an MOI of 0 (no virus). 


\section{3-(4,5-Dimethylthiazol-2-yl)-2,5-diphenyltetrazolium bromide (MTT) assay}

Tumor cells were seeded and infected with VVs similar to the crystal violet staining assay. After a 72-hour incubation, supernatants were removed and $150 \mu \mathrm{L}$ of diluted MTT solution (Cat\# IM0280, Solarbio; Beijing, China) was added to each well at a final concentration of $0.5 \mathrm{mg} / \mathrm{mL}$. After a 4-hour incubation, the supernatants were removed and the formazan crystals were dissolved in $150 \mu \mathrm{L}$ of isopropanol. The OD value was measured at $570 \mathrm{~nm}$ using an absorbance microplate reader. The cell viability was calculated according to the following formula: cell viability $(\%)=\left(\mathrm{OD}_{\text {Treatment }}-\mathrm{OD}_{\text {Blank }}\right) /$ $\left(\mathrm{OD}_{\text {Control }}-\mathrm{OD}_{\text {Blank }}\right) \times 100 \%$.

\section{Cell Counting Kit-8 (CCK8) assay}

H22 cells were seeded at a density of $1 \times 10^{4}$ cells per well and infected with VVs similar to the crystal violet staining assay. After a 72-hour incubation, $10 \mu \mathrm{L}$ of CCK-8 solution (Cat\# C0037, Beyotime) was added to each well of the plate. After a 1-hour incubation, the OD value was measured at $450 \mathrm{~nm}$, and the cell viability is calculated similarly to the MTT assay.

\section{Viral replication}

MC38, CT26, and 4T1 cells were seeded in 24-well plates at $5 \times 10^{4}$ cells per well and placed in an incubator at $37^{\circ} \mathrm{C}$ with a $5 \% \mathrm{CO}_{2}$ atmosphere. When the cells were grown to $>90 \%$ confluency, VVs were added to the wells at an MOI of 0.1 . The seeding of the H22 cells was the same as previously describe and immediately infected with VVs at an MOI of 1. After culturing for 12, 24, 48, 72, and 96 hours, $500 \mu \mathrm{L}$ of proteinase K lysis buffer (containing $100 \mu \mathrm{g} / \mathrm{mL}$ proteinase $\mathrm{K}, 50 \mathrm{mmol}$ potassium chloride, $10 \mathrm{mmol}$ Tris, $0.5 \%$ Tween) was added to each well, and the cell lysates were harvested by pipetting repeatedly. The cell lysates were heated at $56^{\circ} \mathrm{C}$ for $45 \mathrm{~min}$ to completely lyse the tumor cells and to release the viral genomic DNA. Subsequently, the prepared DNA templates were subjected to a standard quantitative PCR (qPCR amplification) using the AceQ Universal SYBR qPCR Master Mix (Cat\# Q511, Vazyme, Nanjing, China) and primers specific to the secreted epidermal growth factor-like (C11R) gene of VV (forward, 5'-AAACACACACTGAGAAACAGCATAAA-3'; reverse, 5'-ACTCGGCGAATGATCTGATTA-3') on a ViiA 7 Real-Time PCR System (Applied Biosystems-Thermo Fisher Scientific, USA). After amplification, the CT value of each time point was obtained and the CT value of the 12 hours was subtracted to obtain the $\Delta \mathrm{CT}$. The replication multiple of the virus at each time point is calculated by $2^{(-\Delta \mathrm{CT})}$.

Viral replication was also measured by virus titer. Briefly, tumor cells were seeded and infected as previously described. The cells were harvested after 12, 24, 48, 72 , and 96 hours, then lysed by three freeze-thaw cycles, and centrifuged at $3000 \times \mathrm{g}$ for $10 \mathrm{~min}$. The supernatants were harvested and the virus titer was determined by a
TCID50 method. The fold change was calculated relative to the virus titer at 12 hours.

\section{Animal experiments}

$\mathrm{BALB} / \mathrm{c}$ mice and C57BL/6 mice were purchased from the Model Animal Research Center of Nanjing University (Nanjing, China). For the establishment of subcutaneous tumor models, the 4T1, MC38, CT26, or H22 tumor cells were implanted subcutaneously into the right flank of the mice. When the tumor reached approximately 50 or 100 $\mathrm{mm}^{3}$, the mice were randomly grouped and intratumorally treated with VVs or phosphate-buffered saline (PBS). Tumor diameters were measured every 2 days using a vernier caliper and the tumor volume was calculated by the formula $0.5 \times$ length $\times$ width. ${ }^{2}$ When the tumor volume reached $2000 \mathrm{~mm}^{3}$, mice were euthanized. For the establishment of the hepatocellular carcinoma ascites model, H22 cells were injected into the peritoneal cavity of the mice. When ascites formed (peritoneal paracentesis was used to confirm the formation of ascites), the mice were randomly grouped and intraperitoneally treated with VVs or PBS. Ascites cells were obtained by paracentesis from the peritoneal cavity of the tumor-bearing mice and flow cytometry was used to measure the proportion of tumor cells in the ascites.

For depletion of $\mathrm{CD}^{+} \mathrm{T}$ or NK cells, each mouse was injected intraperitoneally with $500 \mu \mathrm{g}$ of anti-CD8 $\alpha$ antibody (Clone YTS 169.4, Cat\# BP0117, BioXCell, USA) or anti-NK1.1 antibody (Clone PK136, Cat\# BP0036, BioXCell). Flow cytometry was used to check whether the in vivo depletion was successful.

For the combination therapy with VV-scFv-TIGIT and antimouse PD-1 antibody ( $\alpha$ PD1, Clone RMP1-14, Cat\# BE0146, BioXCell), subcutaneous MC38, and CT26 models were established as previously described. Each mouse was injected intraperitoneally with $200 \mu$ g of $\alpha$ PD1, which was initiated from the beginning of viral treatment and continued 6 or 7 times every 2 days. For the combination therapy with VV-scFv-TIGIT and antimouse LAG-3 antibody ( $\alpha$ LAG-3, Clone C9B7W, Cat\# BE0174, BioXCell), subcutaneous CT26 model was established as previously described. Each mouse was injected intraperitoneally with $200 \mu \mathrm{g}$ of $\alpha \mathrm{LAG}-3$, which was initiated 2 days post the first viral injection and continued 3 times every 4 days.

\section{Extracellular and intracellular staining and flow cytometry}

The follows antibodies were purchased from BioLegend (California, USA): phycoerythrin (PE) antimouse PD-L1 (Clone 10F.9G2, Cat\# 124308), Allophycocyanin (APC) antimouse CD45 (Clone 30-F11, Cat\# 103112), fluorescein-5-isothiocyanate (FITC) or PE antimouse CD3 (Clone 17A2, Cat\# 100204; Cat\# 100206), FITC or PE antimouse CD4 (Clone GK1.5, Cat\# 100406; Cat\# 100408), peridinin-chlorophyll-protein-cyanine5.5 (PerCP-Cy5.5) antimouse CD8 $\alpha$ (Clone 53-6.7, Cat\# 100734), PE antimouse CD49b (Clone HM $\alpha 2$, Cat\# 103520), PE antimouse TIGIT (Clone 1G9, Cat\# 142104), 
PE antimouse PD-1 (Clone 29F.1A12, Cat\# 135206), PE antimouse TIM-3 (Clone B8.2C12, Cat\# 134004), PE antimouse LAG-3 (Clone C9B7W, Cat\# 125208), FITC antimouse CD107a (LAMP-1; Clone 1D4B, Cat\# 121606), $\mathrm{PE}$ antimouse/rat tumor necrosis factor (TNF)- $\alpha$ (Clone MP6-XT22, Cat\# 506306), PE antihuman/mouse granzyme B (Clone QA18A28, Cat\# 396406), FITC antimouse CD62L (Clone MEL-14, Cat\# 104406), PE antimouse/ human CD44 (Clone IM7, Cat\# 103024) and PE rat IgG2b ( $\kappa$ isotype control; Clone RTK4530, Cat\# 400607). FITC antimouse NK1.1 (Clone PK136, Cat\# 11-5941-82) was purchased from eBioscience-Thermo Fisher Scientific (USA).

For the preparation of single-cell suspensions of tumor tissues, mice were anesthetized and sacrificed, and the tumor tissues were harvested and placed in a serum-free medium (Cat\# H740KJ, Basalmedia) with $0.2 \%$ collagenase IV (Cat\# C4-22-1G, Sigma-Aldrich, Germany). Then, the tumor tissues were cut into $1-2 \mathrm{~mm}$ pieces, digested for 2 hours, and passed through $70 \mu \mathrm{m}$ nylon filters (Cat\# CSS013070, Jetbiofil, Guangzhou, China) to obtain the single-cell suspensions. After that, the collagenase was removed by centrifugation, and the cell pellets were suspended in the serum-free medium and adjusted to $2 \times 10^{7}$ cells $/ \mathrm{mL}$. For the preparation of splenocytes, the spleens were obtained from the sacrificed mice and ground into single-cell suspensions using syringe plungers on $70 \mu \mathrm{m}$ nylon filters (Cat\# CSS013070, Jetbiofil). Then, the cells were counted and adjusted to $5 \times 10^{6}$ cells $/ \mathrm{mL}$. Ascites cells were obtained by paracentesis from the peritoneal cavity of the tumor-bearing mice. After cell counting, the cells were adjusted to $2 \times 10^{7}$ cells $/ \mathrm{mL}$. For tumor cell lines, the adherent cells (4T1, MC38, CT26) were digested with $0.5 \%$ trypsin in advance, and the suspension-cultured H22 cells were directly adjusted to $2 \times 10^{6}$ cells $/ \mathrm{mL}$. For extracellular staining, the preprepared single-cell suspensions were incubated with the fluorescent monoclonal antibodies for $15 \mathrm{~min}$ at RT. After incubation, the cells were fixed with 4\% (w/v) paraformaldehyde (PFA, Cat\# G1101, Servicebio, Wuhan, China) solution and directly analyzed using a FACS Calibur cytometer (BD; California, USA). For intracellular staining, the fixed cells were ruptured with $1 \times$ permeabilization buffer (Cat\# 00-8333-56, eBioscience), and then the corresponding antibodies were added respectively and incubated for $30 \mathrm{~min}$ in the dark. After washing with PBS once, an appropriate amount of PBS was added to resuspend the cells and immediately analyzed by flow cytometry. Data analysis was performed using FlowJo software (TreeStar; OR, USA).

\section{Measurement of cytokines}

Ascites fluids were collected by paracentesis from the peritoneal cavity of the mice. Samples were centrifuged at $600 \mathrm{~g}$ for $5 \mathrm{~min}$ at RT and the supernatants were collected. ELISA MAX Standard Sets of IFN- $\gamma$ (Cat\# 430801, Biolegend), IL-2 (Cat\# 431001), IL-6 (Cat\# 431301), and IL-10 (Cat\# 431411) were used to determine the concentration of the corresponding cytokines. The measurement method was based on the manufacturing protocol.

For co-culture assay, C57BL/6 mice cured of H22 by VV-scFv-TIGIT were re-inoculated with $2 \times 10^{6} \mathrm{H} 22$ cells intraperitoneally. The re-inoculation was performed once a month and a total of two inoculations. Seven days after the second intraperitoneal inoculation of $\mathrm{H} 22$ cells, the mice were sacrificed after anesthesia to obtain spleens and prepare splenocytes. The cultured H22 cells were seeded into a 6 -well plate at the density of $3 \times 10^{5}$ cells per well, and then $3 \times 10^{6}$ splenocytes were added to make the ratio of splenocytes to tumor cells 10:1. The cells were harvested after 72 hours of co-culture. The preparation of the supernatants and the measurement of cytokines were performed as previously described.

\section{Immunohistochemistry}

The resected tumor tissues were fixed in 4\% PFA (Cat\# G1101, Servicebio) and embedded in paraffin blocks. Sections were cut at a thickness of $5 \mu \mathrm{m}$ from the paraffinembedded blocks, deparaffinized by immersion in xylene $(10 \mathrm{~min}, \times 2)$, and rehydrated in graded ethanol $(100 \%$ for $3 \mathrm{~min}, \times 2$; $95 \%$ for $3 \mathrm{~min} ; 70 \%$ for $3 \mathrm{~min}$; $50 \%$ for $3 \mathrm{~min})$ and incubated with $3 \%(\mathrm{w} / \mathrm{v})$ hydrogen peroxide to block endogenous peroxidase activity. After that, the sections were incubated with a rabbit antimouse $\mathrm{CD} 8 \alpha$ antibody (1:200 dilution; Cat\# ab217344, Abcam, USA) and an HRP goat antirabbit IgG secondary antibody (1:200 dilution; Cat\# G1213, Servicebio). Finally, the sections were stained with 3',3-diaminobenzidine (Cat\# G1212-2, Servicebio) and counterstained with 37\% (w/v) hematoxylin (Cat\# G1004, Servicebio).

\section{Statistical analyses}

All Statistical analyses were performed using Prism 8.2.1 (GraphPad Software, California, USA). The statistical differences among the groups were analyzed by analysis of variance. The Kaplan-Meier method was used to draw the survival curve, and the Log-Rank test was used to calculate the statistical significance among the groups. In all statistical analyses, $\mathrm{p}<0.05$ was considered statistically significant.

\section{RESULTS}

\section{Generation and characterization of recombinant oncolytic VVs}

Through homologous recombination, we generated a recombinant oncolytic VV, VV-scFv-TIGIT, with a gene fragment of $\mathrm{scFv}$ against TIGIT in the backbone of a thymidine kinase (TK; J2R)-destroyed WR-VV (figure 1A). We also generated a control VV, VV-control, with no scFv insertion in the TK area. The VV-scFv-TIGIT virus exhibited green fluorescent plaques in Hela-S3 cells, allowing it to be easily picked and purified as a monoclonal virus (figure 1B). The scFv-TIGIT was efficiently secreted from VV-scFv-TIGIT-infected Hela-S3 cells as detected by western blot assay (figure 1C). Similarly, four murine 

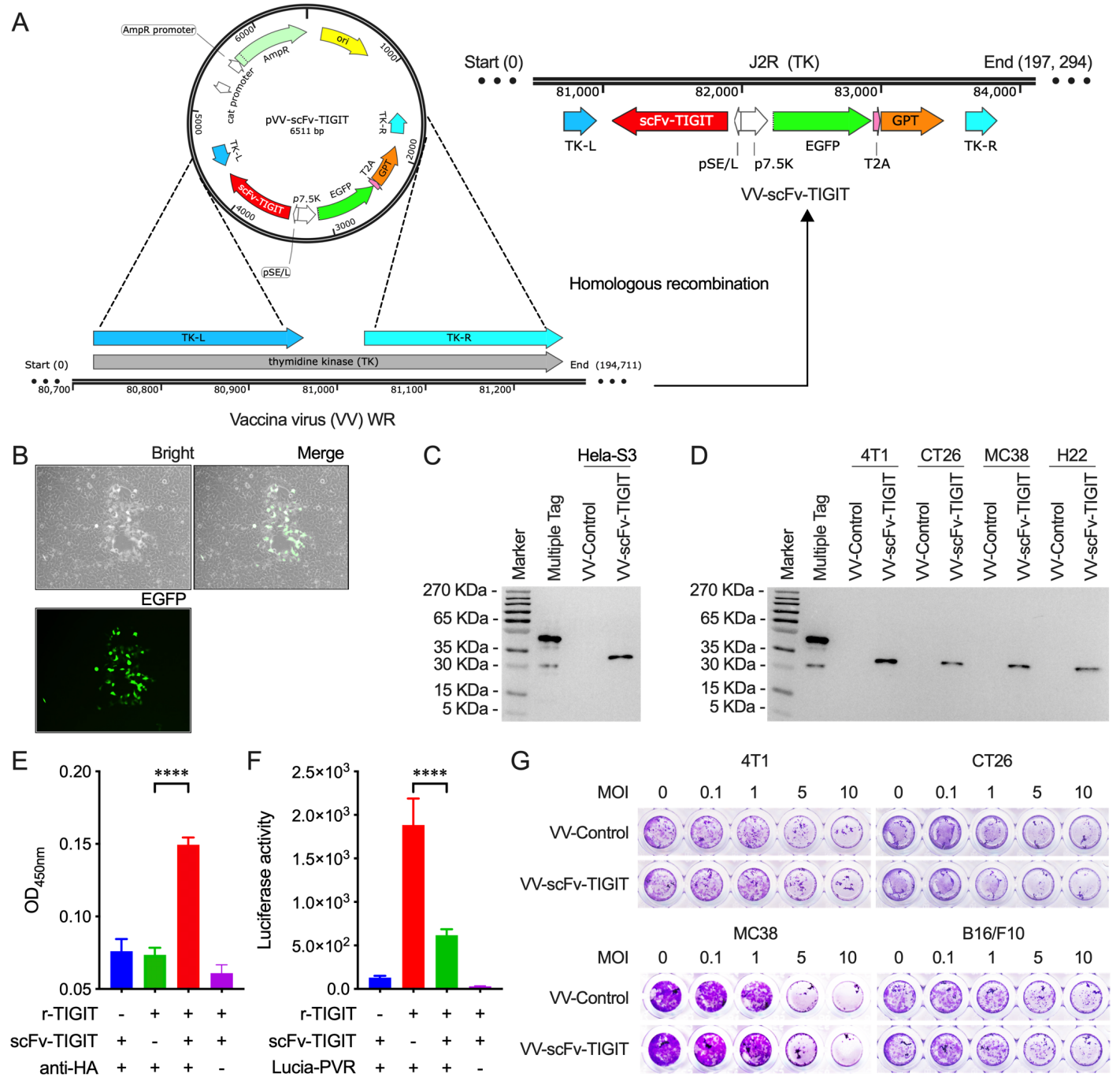

G

$4 \mathrm{~T} 1$
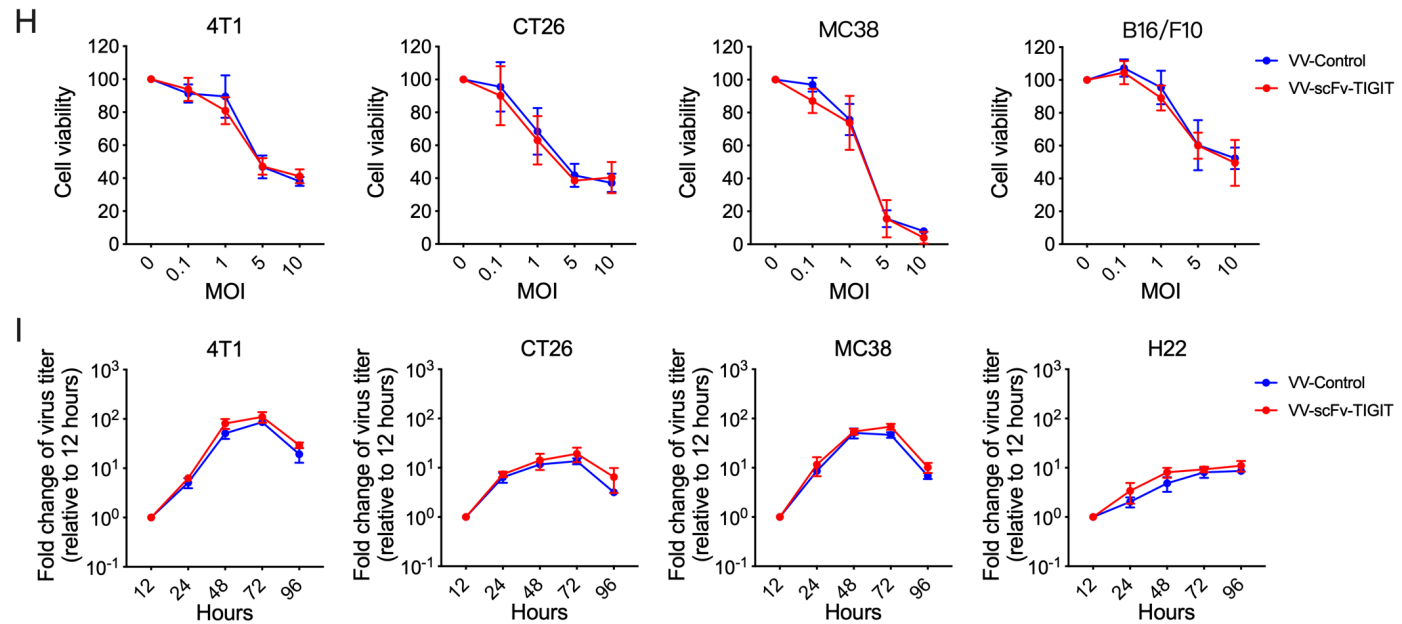

Figure 1 Generation and characterization of VV-scFV-TIGIT. (A) A schematic diagram of homologous recombination. To generate VV-scFv-TIGIT, the shuttle plasmid pVV-scFV-TIGIT was used for homologous recombination with a western reserve (WR) strain of $\mathrm{V} V$ by using the left (L) and right (R) flanking sequences of thymidine kinase (TK). T2A, thoseaasigna virus $2 \mathrm{~A}$. (B) The virus plaque formed in Hela-S3 cells infected with VV-scFv-TIGIT. (C) Western blot analysis of HA-tagged scFv-TIGIT in the supernatants of Hela-S3 cells, a Multiple Tag was used as the positive control. (D) Western blot analysis of scFv-TIGIT in four murine tumor cell lines. (E) ELISA was used to detect the binding of the secreted scFv-TIGIT to the recombinant TIGIT (r-TIGIT). (F) Luciferase-linked immunosorbent assay was used to test the blocking effect of scFv-TIGIT on the binding of PVR and r-TIGIT. (G, H) Crystal violet staining was used to detect the oncolytic ability of VVs against murine tumor cells. (I) TCID50 method was used to detect viral replication in murine tumor cells. ${ }^{* t * t} \mathrm{p}<0.0001$. scFv, single-chain variable fragment; TIGIT, T-cell immunoglobulin and ITIM domain; VV, vaccinia virus. 
tumor cells (4T1, MC38, CT26, and H22) infected with VV-scFv-TIGIT also efficiently secreted the scFv-TIGIT (figure 1D). ELISA and luciferase assay showed that the secreted scFv-TIGIT effectively bound to r-TIGIT (figure 1E), and effectively blocked the binding of PVR to r-TIGIT (figure 1F). A crystal violet staining assay showed that both VV-scFv-TIGIT and VV-Control showed dose (MOI)-dependent oncolytic activity against 4T1, MC38, CT26, and B16/F10 cells (figure 1G). Both crystal violet staining and MTT assay confirmed that there was no statistical difference in the oncolytic activity between $\mathrm{VV}-\mathrm{scF}$ TIGIT and VV-Control (figure 1H; online supplemental figure S2A). Moreover, VV-scFv-TIGIT and VV-Control showed similar replication characteristics in murine tumor cell lines (figure 1I; online supplemental figure S2B). These data indicated that the replication and oncolytic ability of the recombinant $\mathrm{VV}$ was not affected by the scFv-TIGIT transgene.

\section{Antitumor activity of VVs on subcutaneous tumor models}

We evaluated the antitumor activity of the VVs in four subcutaneous tumor models (4T1, CT26, H22 (figure 2A), and MC38 (figure 2B)) in immunocompetent BALB/c (figure 2A) and C57BL/6 mice (figure 2B). Although the administration of VV-Control showed superior antitumor effects than PBS in the CT26 model in terms of inhibiting tumor growth and prolonging the survival of mice, it did not show any superior antitumor effects than PBS in the 4T1, H22, and MC38 models. In all four models, mice treated with VV-scFv-TIGIT had significantly lower tumor volume and prolonged survival compared with mice treated with VV-Control or PBS (figure 2A,B). Of note, nearly $20 \%$ of the CT26 tumor-bearing mice treated with VV-scFv-TIGIT achieved complete remission (CR), while none of the mice treated with VV-Control or PBS achieved CR (figure 2A; online supplemental figure S3). There was no significant difference in body weight among the three groups of mice (figure 2A,B). These data indicated that VV-scFv-TIGIT is superior to VV-Control in reducing tumor burden and prolonging the survival of tumor-bearing mice.

\section{Intratumoral injection of VVs induced infiltration of immune cells}

We evaluated the infiltration of immune cells after intratumoral injection of the VVs in the CT26 subcutaneous tumor model (figure 3A). Consistent with the previous results, administration of VV-scFv-TIGIT significantly delayed the tumor growth ( $\mathrm{p}<0.05$ vs VV-Control, $\mathrm{p}<0.01$ vs PBS; figure $3 \mathrm{~B}, \mathrm{C})$, but it did not affect the body weight of the mice $(p>0.05$; figure 3D). Flow cytometry analysis showed that intratumoral injection of VV-scFv-TIGIT significantly increased the infiltration of $\mathrm{CD}_{4} 5^{+}$lymphocytes, $\mathrm{CD} 3^{+} \mathrm{T}, \mathrm{CD} 4^{+} \mathrm{T}$, and $\mathrm{CD}^{+} \mathrm{T}$ cells compared with either VV-Control or PBS, but did not cause obvious changes in NK and NKT cells (figure 3E,F). Next, we evaluated the expression of four immune checkpoints (TIGIT, PD-1, TIM-3, and LAG-3) and one activation marker (CD107A) on T cells (figure 3G-I). Compared with PBS, injection of VV-Control only increased the composition of TIGIT $^{+}$cells in the $\mathrm{CD}^{+} \mathrm{T}$ cells, and increased the composition of $\mathrm{TIGIT}^{+}$and LAG-3 $3^{+}$cells in the $\mathrm{CD}^{+}{ }^{+} \mathrm{T}$ cells, whereas injection of VV-scFv-TIGIT significantly increased the composition of TIGIT $^{+}$, PD- $^{+}$, TIM-3 ${ }^{+}$, LAG $^{+}{ }^{+}$and CD $107 \mathrm{~A}^{+}$ cells in the $\mathrm{CD}^{+} \mathrm{T}$ cells and increased the composition of TIGIT $^{+}$and LAG $-3^{+}$cells in the $\mathrm{CD} 4^{+} \mathrm{T}$ cells. In particular, the composition of $\mathrm{LAG}-3^{+}$and $\mathrm{CD} 107 \mathrm{~A}^{+}$cells in the $\mathrm{CD} 8^{+} \mathrm{T}$ cells were significantly different between the VV-scFv-TIGIT and V-Control injection groups $(\mathrm{p}<0.05 ; \mathrm{p}<0.01)$. Moreover, VV-scFv-TIGIT significantly increased the proportion of $\mathrm{CD}^{+} \mathrm{T}$ cells in the splenocytes ( $\mathrm{p}<0.05 \mathrm{vs}$ PBS; figure $3 \mathrm{~J}$ ). Subsequently, we confirmed the infiltration of $\mathrm{CD}^{+} \mathrm{T}$ cells by immunohistochemistry. Consistent with the flow cytometry analysis, VV-scFv-TIGIT significantly increased the tumor infiltration of $\mathrm{CD}^{+} \mathrm{T}$ cells compared with either VV-Control or PBS ( $\mathrm{p}<0.001 ; \mathrm{p}<0.0001$; online supplemental figure $\mathrm{S} 4$ ). As expected, comparable virus titers were observed in tumors of mice treated with VV-scFv-TIGIT or VV-Control, whereas no virus was detected in the tumor tissues of PBS-treated mice and in the sera of the three groups of mice (figure 3K). Altogether, these results indicated that the VV-scFv-TIGIT could reshape the TME by recruiting immune cells and activating the tumor-infiltrating $\mathrm{CD}^{+} \mathrm{T}$ cells.

\section{Enhanced antitumor immunity in the ascites tumor models}

We evaluated the ability of VV-scFv-TIGIT to recruit immune cells and its antitumor activity on an H22 ascites model in C57BL/6 mice (online supplemental figure S5A; figure 4A). Through dynamic observation, we found that tumor cells and lymphocytes were always maintained in a fixed ratio in the ascites of mice treated with PBS, showing a 'cold' TME lacking lymphocytes (approximately 1\%). However, intraperitoneal injection of VV-Control or VV-scFv-TIGIT gradually reduced the proportion of tumor cells, while gradually increased the proportion of $\mathrm{CD} 45^{+}$lymphocytes (online supplemental figure S5B,C), which made the TME become 'hot'. Of note, the tumor cells in the ascites of VV-scFvTIGIT-treated mice almost disappeared (2.09\%) 2 days post the third VV treatment, accompanied by a higher proportion $(25.2 \%)$ of lymphocytes (online supplemental figure S5B,C). Two days post the second VV treatment, VV-scFvTIGIT-treated mice had a significantly lower proportion of tumor cells and a higher proportion of lymphocytes, NK, $\mathrm{CD}^{+} \mathrm{T}, \mathrm{CD}^{+} \mathrm{T}$, and $\mathrm{CD}^{+} \mathrm{T}$ cells in the ascites compared with PBS-treated or VV-Control-treated mice (figure 4B). Both VV-scFv-TIGIT and VV-Control significantly increased the composition of TIGIT $^{+}$, PD- $1^{+}$, TIM- $3^{+}$, and LAG- $3^{+}$cells in the $\mathrm{CD}^{+} \mathrm{T}$ population compared with PBS. Of note, VV-scFv-TIGIT was more potent in increasing the proportion of TIM- $3^{+} \mathrm{CD} 8^{+}$, and LAG- $3^{+} \mathrm{CD} 8^{+} \mathrm{T}$ cells than VV-Control (figure 4C). Moreover, VV-scFv-TIGIT was shown to activate $\mathrm{CD}^{+} \mathrm{T}$ cells more effectively than VV-Control, which is mainly reflected in the increased expression of CD107a on the cell surface, and the increased expression of intracellular IFN- $\gamma$, TNF- $\alpha$, and granzyme B (figure 4D). Mice treated with VV-scFv-TIGIT also showed a higher level of IFN- $\gamma$, IL-2, IL-6, and IL-10 in ascites than mice treated with VV-Control or PBS (figure $4 \mathrm{E})$. Consistent with these results, $>90 \%(15 / 16)$ of 
A
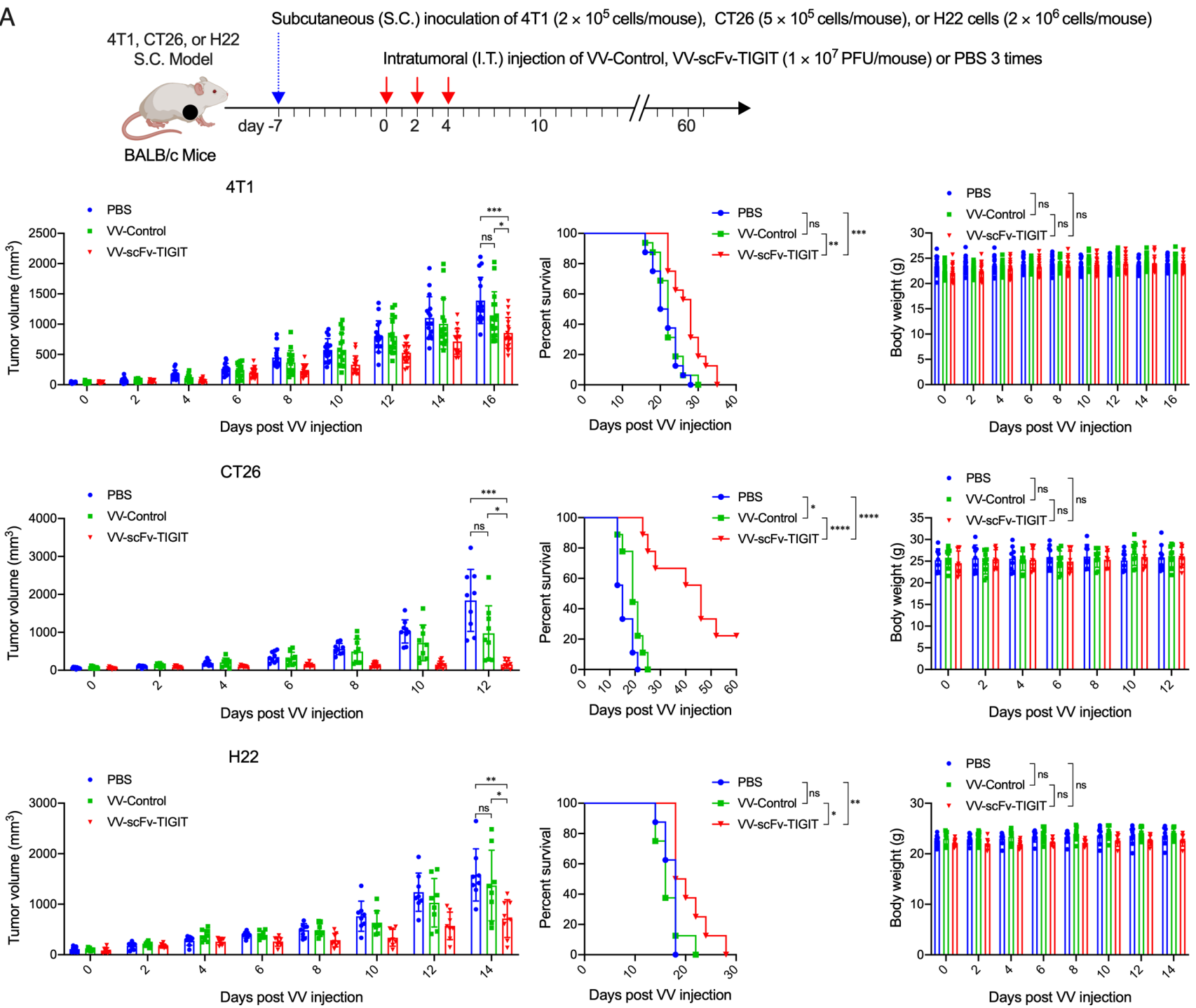

B
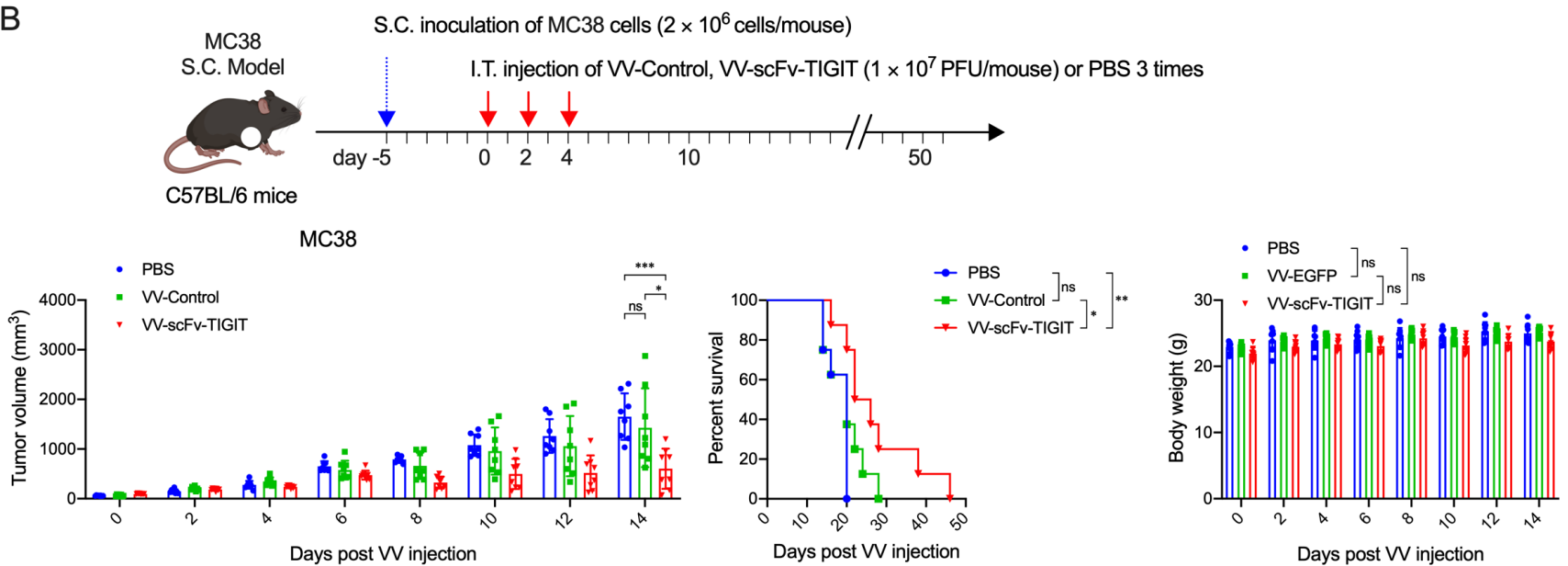

Figure 2 Intratumoral injection of VV-scFv-TIGIT enhanced antitumor efficacy in subcutaneous (S.C.) tumor models. (A) The S.C. tumor models of $4 \mathrm{~T} 1, \mathrm{CT} 26$, and H22 were established by inoculation of corresponding cells on the right flank of BALB/C mice. Seven days after tumor inoculation, mice were administered intratumorally (I.T.) with the indicated VV or PBS. (B) MC38 S.C. tumor model was similarly established on C57BL/6 mice and injected intratumorally with the indicated VV or PBS 5 days after the tumor inoculation. Tumor volume and body weight were measured every 2 days. Error bars represent SD. Once the tumor volume exceeded $2000 \mathrm{~mm}^{3}$, the mouse was considered dead. ns, no significant differences; ${ }^{*} p<0.05$; ${ }^{* *} p<0.01$; ${ }^{* \star *} p<0.001 ;{ }^{* * \star *} p<0.0001$. PBS, phosphate-buffered saline; scFv, single-chain variable fragment; TIGIT, T-cell immunoglobulin and ITIM domain; VV, vaccinia virus. 
A
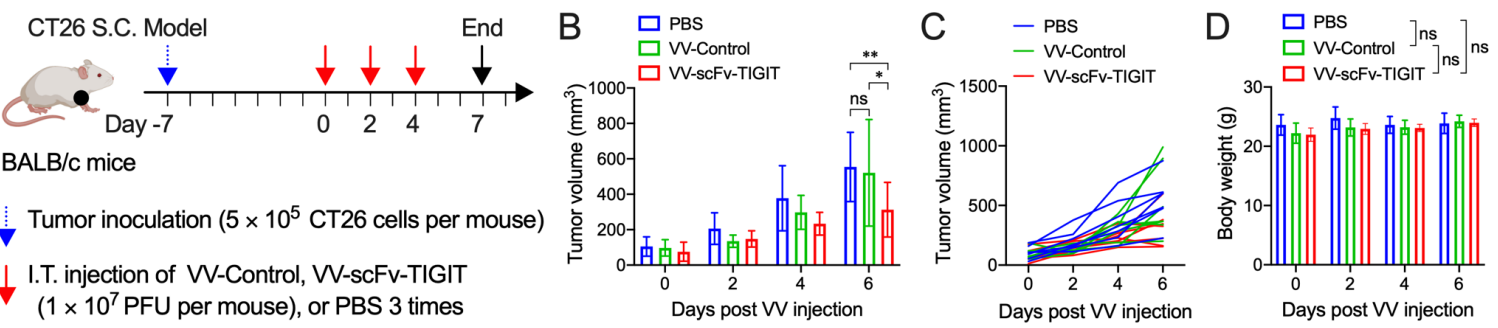

E
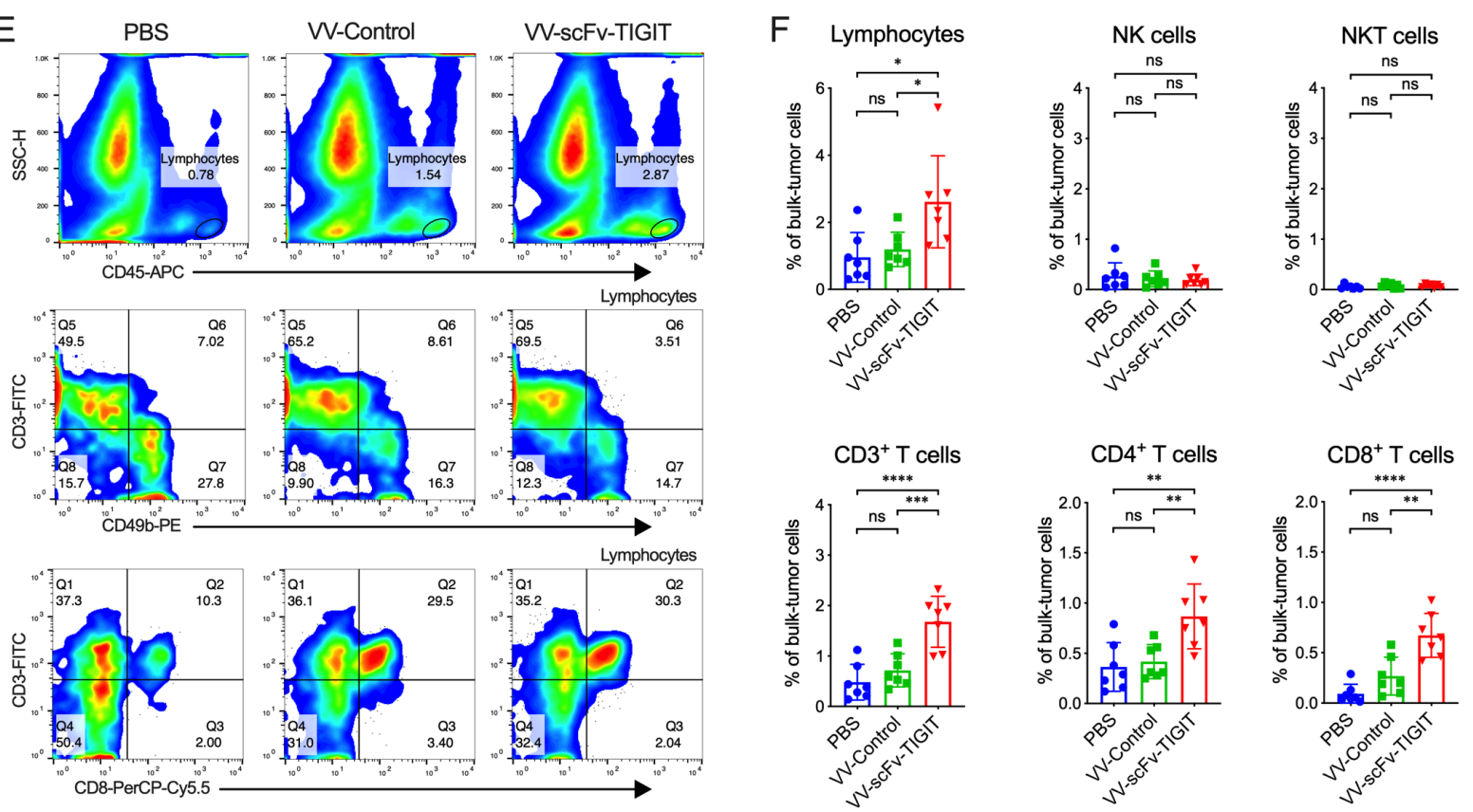

G
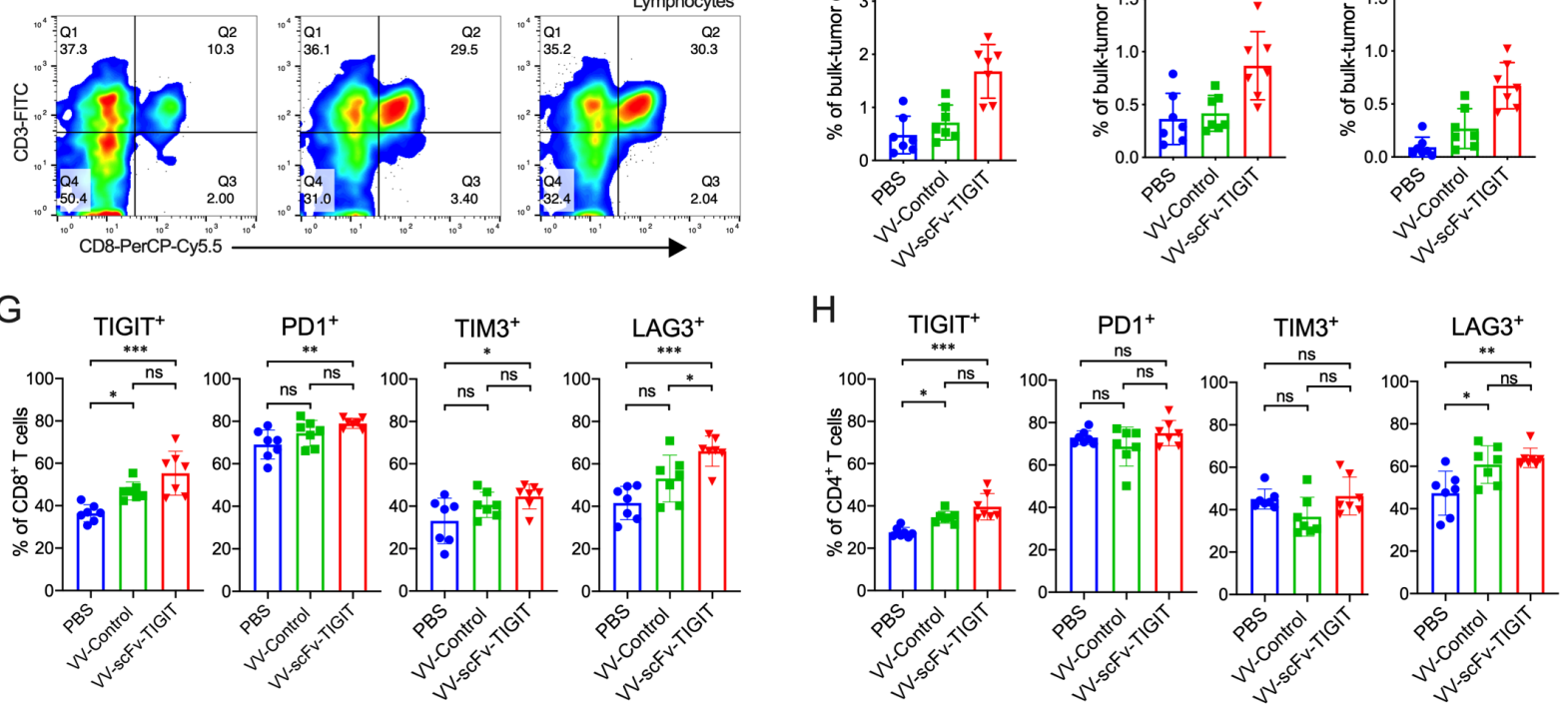

I

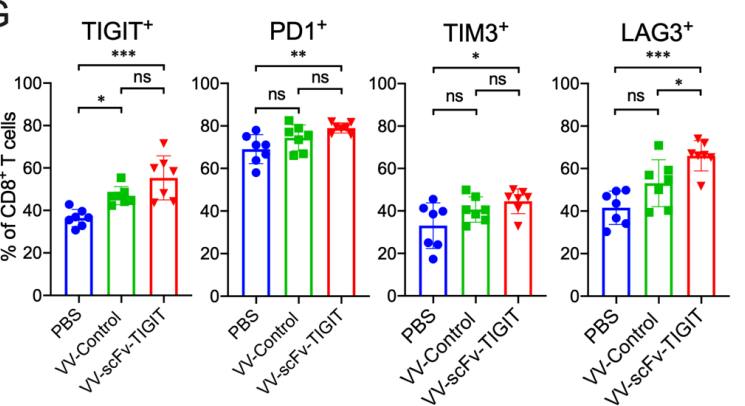

$\mathrm{K}$

Tumor

Sera
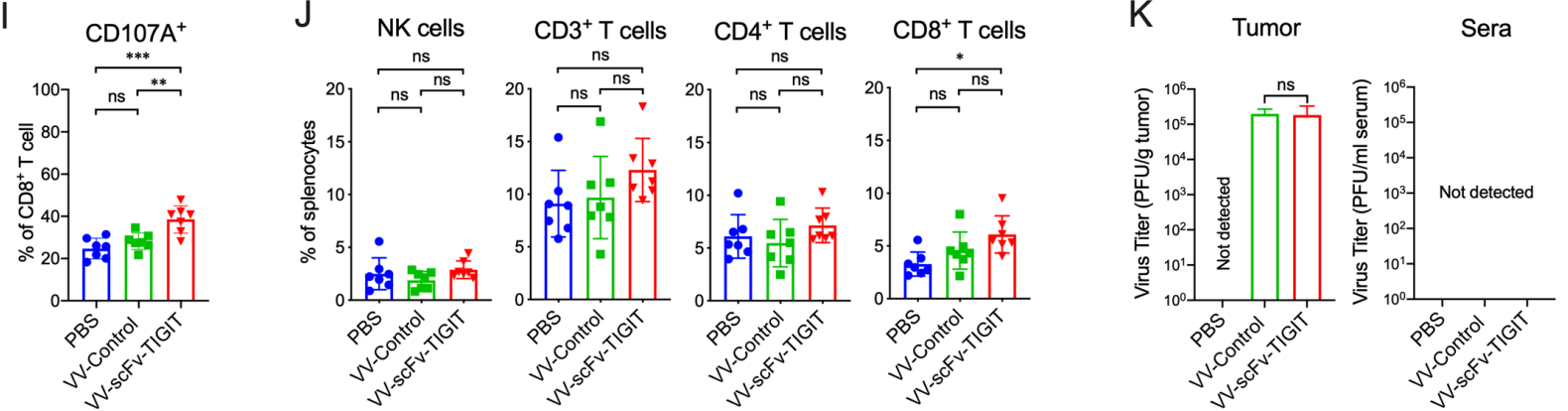

Figure 3 Intratumoral injection of VV-scFv-TIGIT enhanced infiltration of immune cells in the CT26 tumor model. (A) Treatment scheme of CT26 subcutaneous (S.C.) tumor model. (B) Mean tumor volume of mice. (C) Individual tumor growth curve of mice. (D) Body weight of mice. (E) Representative diagram of flow cytometric analysis of immune cell infiltration in tumor tissues. (F) Flow cytometric analysis of the proportion of lymphocytes and their subpopulations in tumor tissues. (G) Flow cytometric analysis of the expression of immune checkpoints on $\mathrm{CD}^{+} \mathrm{T}$ cells. $(\mathrm{H})$ Flow cytometric analysis of the expression of immune checkpoints on $\mathrm{CD}^{+} \mathrm{T}$ cells. (I) Flow cytometric analysis of the expression of CD107A on CD8 ${ }^{+} \mathrm{T}$ cells. (J) Flow cytometric analysis of the proportion of lymphocytes subsets in splenocytes. (K) The virus titers in tumor tissues or sera of mice were quantified by the TCID50 method. ns, no significant differences; ${ }^{*} p<0.05 ;{ }^{* *} p<0.01 ;{ }^{* * *} p<0.001 ;{ }^{* * *} p<0.0001$. PBS, phosphatebuffered saline; scFv, single-chain variable fragment; TIGIT, T-cell immunoglobulin and ITIM domain; VV, vaccinia virus. 
A

Intraperitoneal inoculation of $\mathrm{H} 22$ cells $\left(1 \times 10^{7}\right.$ cells/mouse $)$

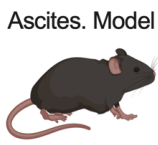

C57/BL6 mice
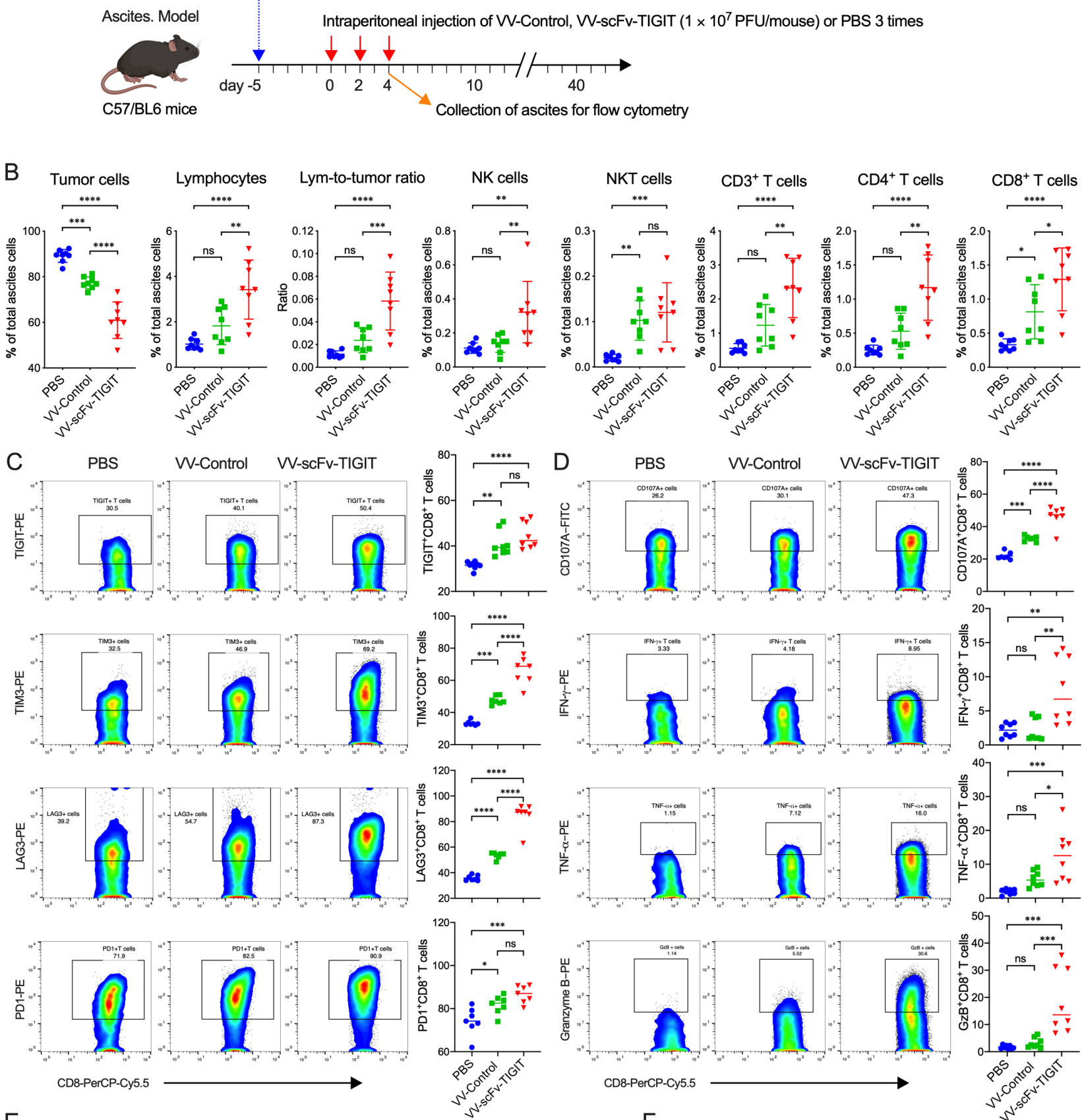

E
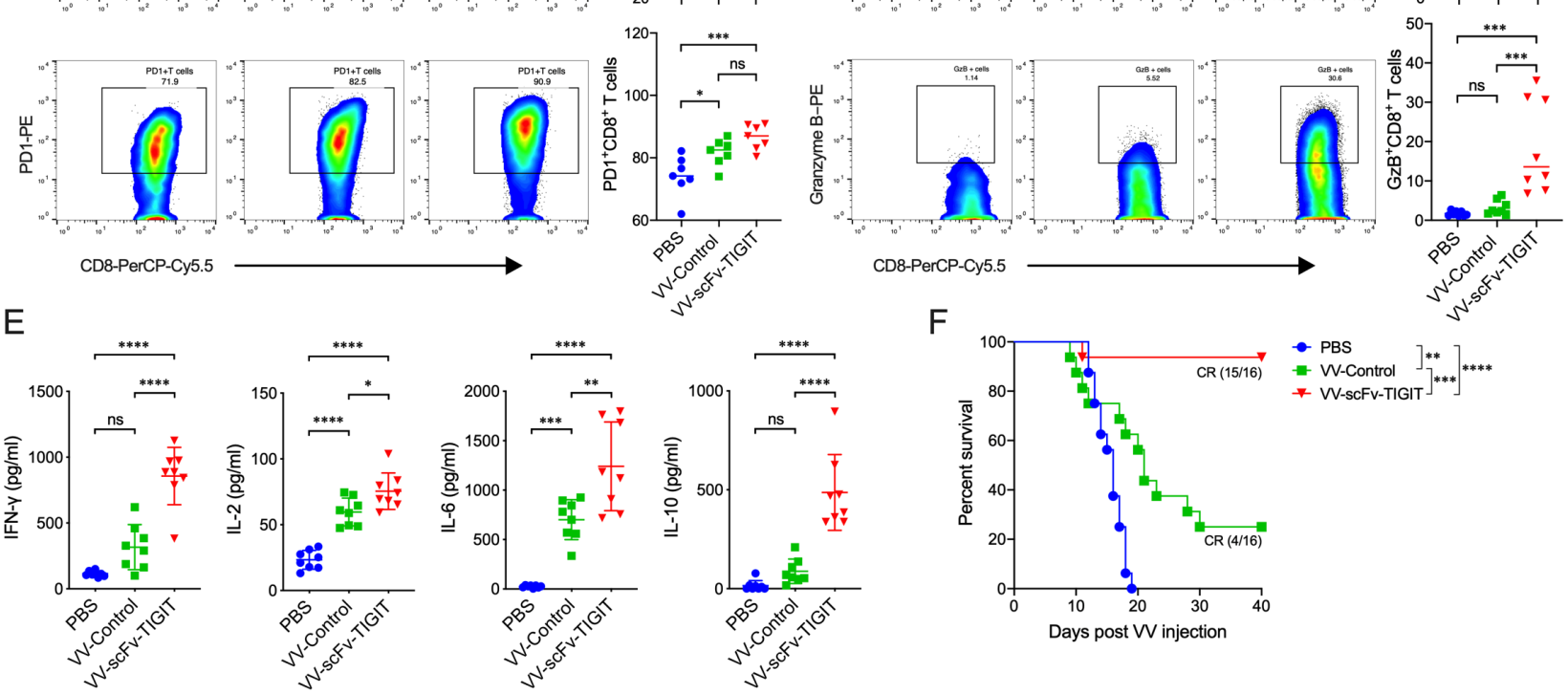

Figure 4 Intraperitoneal injection of VV-scFV-TIGIT enhanced antitumor efficacy in ascites tumor models. (A) Treatment scheme of H22 ascites model. (B) Flow cytometric analysis of the proportion of tumor cells, lymphocytes, and their subpopulations in tumor tissues. (C) Flow cytometric analysis of the expression of immune checkpoint molecules on the surface of $\mathrm{CD}^{+} \mathrm{T}$ cells. (D) Surface and intracellular staining of $\mathrm{CD} 8^{+} \mathrm{T}$ cells for $\mathrm{T}$ cell activation markers and analysis by flow cytometry. (E) The levels of cytokines in the ascites were detected by ELISA. (F) Kaplan-Meier survival curves of tumor-bearing mice. ns, no significant differences; ${ }^{\star} p<0.05 ;{ }^{* *} p<0.01 ;{ }^{* * *} p<0.001 ;{ }^{* \star *} p<0.0001$. PBS, phosphate-buffered saline; scFv, single-chain variable fragment; TIGIT, T-cell immunoglobulin and ITIM domain; VV, vaccinia virus. 
mice treated with VV-scFv-TIGIT achieved CR of ascites, while only $25 \%(4 / 16)$ of mice treated with VV-Control achieved CR (figure $4 \mathrm{~F}$ ).

We further evaluated the antitumor activity of $\mathrm{VV}-\mathrm{scFv}$ TIGIT on another H22 ascites model in BALB/c mice (online supplemental figure S6A). Consistent with previous results, intraperitoneal injection of VV-scFv-TIGIT significantly increased the proportion of lymphocytes $(p<0.05 \mathrm{vs}$ V-Control; $\mathrm{p}<0.001$ vs PBS), CD4 $4^{+} \mathrm{T}$ cells $(\mathrm{p}<0.01$ vs PBS), and $\mathrm{CD}^{+} \mathrm{T}$ cells $(\mathrm{p}<0.01$ vs VV-Control; $\mathrm{p}<0.0001$ vs PBS $)$ in the ascites (online supplemental figure S6B,C). Nearly $40 \%$ (3/8) of the mice treated with VV-scFv-TIGIT achieved CR of ascites, while other mice showed continuous ascites progression and eventually died (online supplemental figure S6D). These data, together with the previous data, indicated that VV-scFv-TIGIT had superior antitumor efficacy and a stronger ability to recruit and activate $\mathrm{CD} 8^{+} \mathrm{T}$ cells in ascites models.

\section{$\mathrm{CD8}^{+} \mathrm{T}$ cells mediated the antitumor immunity of $\mathrm{VV}-\mathrm{scFv}$ - TIGIT}

To reveal the role of $\mathrm{CD}^{+} \mathrm{T}$ and NK cells in mediating the antitumor activity of VV-scFv-TIGIT, we depleted these two types of lymphocytes and analyzed whether their depletion would affect the antitumor efficacy of $\mathrm{VV}$ in the ascites model (figure 5A). As expected, the $\mathrm{CD} 8^{+}$ $\mathrm{T}$ and NK cells in peripheral blood were completely depleted 1 day after the injection of the corresponding monoclonal antibody (figure 5B) and their depletion was maintained for 2 weeks (data not shown). Similarly, these cells in ascites were depleted when the ascites formed (3 days after the antibody injection) (figure 5C). Consistent with previous results, treatment of mice with $\mathrm{VV}-\mathrm{scF}$ TIGIT significantly decreased the proportion of tumor cells and increased the proportion of lymphocytes, NK, NKT, CD $3^{+} \mathrm{T}, \mathrm{CD} 4^{+} \mathrm{T}$, and $\mathrm{CD} 8^{+} \mathrm{T}$ cells in the ascites (figure 5D,E). In contrast, the depletion of $\mathrm{CD}^{+} \mathrm{T}$ cells reversed all these increases, making these cells in $\mathrm{VV}-\mathrm{scFv}$ TIGIT-treated mice reach the same or lower levels as those in PBS-treated mice (figure 5E). Although the depletion of NK cells also significantly decreased the NKT, $\mathrm{CD}^{+}$, $\mathrm{CD}^{+}$, and $\mathrm{CD}^{+} \mathrm{T}$ cells, these cells still showed a higher trend in VV-scFv-TIGIT-treated mice than in PBS-treated mice (figure 5E). Moreover, IFN- $\gamma$, IL-2, IL-6, and IL-10 were significantly reduced in response to the depletion of $\mathrm{CD}^{+} \mathrm{T}$ cells (figure $5 \mathrm{~F}$ ). Consistent with these results, the depletion of $\mathrm{CD}^{+} \mathrm{T}$ cells completely abrogated the antitumor efficacy of VV-scFv-TIGIT, while the depletion of NK cells had no obvious impact (figure 5G). Moreover, all these cured mice did not develop ascites after continuous observation for 180 days (figure $5 \mathrm{G}$ ). These results indicated that $\mathrm{CD}^{+} \mathrm{T}$ cell is a major immune cell type that mediated the antitumor immunity of VV-scFv-TIGIT.

\section{Treatment of mice with VV-scFv-TIGIT established long- term tumor-specific immunological memory and systemic antitumor immunity}

To examine the tumor-specific immunological memory, 10 of 15 mice previously cured of H22 by VV-scFv-TIGIT were twice intraperitoneally and once subcutaneously rechallenged with the same tumor cells, and once subcutaneously challenged with MC38 cells (figure 6A). All age-matched treatment-naïve mice developed cancerous ascites and eventually died after being challenged by $\mathrm{H} 22$ cells in the peritoneum, while all the previously cured mice did not develop ascites after either the first or the second $\mathrm{H} 22$ rechallenge (figure 6B). Similarly, no subcutaneous tumors were formed in these previously cured mice after a subcutaneous rechallenge of H22 cells. However, all these cured mice did not reject MC38 cells and developed subcutaneous tumors (figure 6C). Consistent with these results, the proportion of naive $\left(\mathrm{CD} 62 \mathrm{~L}^{+} \mathrm{CD} 44^{-}\right)$ $\mathrm{CD}^{+} \mathrm{T}$ cells in splenocytes of mice that had rejected $\mathrm{H} 22$ tumor rechallenge decreased $(p<0.0001 \mathrm{vs}$ treatmentnaive mice), while the total $\mathrm{CD} 8^{+} \mathrm{T}$ cells and their central memory $\left(\mathrm{CD} 6 \mathrm{~L}^{+} \mathrm{CD} 44^{+}\right)$and effector memory $\left(\mathrm{CD} 62 \mathrm{LCD}^{-} 4^{+}\right)$subsets increased $(\mathrm{p}<0.0001, \mathrm{p}<0.001$, $\mathrm{p}<0.001$, respectively; figure $6 \mathrm{D}-\mathrm{E})$. Furthermore, co-culture of H22 cells with splenocytes from the H22 rechallenged mice significantly reduced the proportion of the tumor cells and increased the production of IFN- $\gamma$ and IL-2 in the co-culture system (all $\mathrm{p}<0.05 \mathrm{vs}$ treatmentnaïve mice; figure $6 \mathrm{~F}$ ).

Next, to investigate whether intraperitoneal injection of VV-scFv-TIGIT could establish a systemic antitumor immunity against the subcutaneous tumor, we established a mouse dual tumor model by inoculation of the H22 cells intraperitoneally and subcutaneously (online supplemental figure S7A). To our surprise, all the ascites in mice that had been intraperitoneally injected with VV-scFv-TIGIT disappeared (online supplemental figure S7B). Meanwhile, the subcutaneous tumors in 9 of 11 mice also completely regressed (online supplemental figure S7C). In contrast, all of the PBS-treated mice died of ascites (online supplemental figure S7B,E), and none of these mice showed regression of subcutaneous tumors (online supplemental figure S7D,E). Moreover, no virus was detected in the subcutaneous tumors of the mice treated with an intraperitoneal injection of VV-scFv-TIGIT (online supplemental figure S7F). These data indicated that the treatment of tumor-bearing mice with $\mathrm{VV}-\mathrm{scFv}$ TIGIT established long-term tumor-specific immunological memory and systemic antitumor immunity.

\section{Combination therapy with $\alpha \mathrm{PD}-1$ or aLAG-3 improved antitumor efficacy of VV-scFv-TIGIT in colon cancer models}

First, we analyzed the correlation between the expression of TIGIT and the other two immune checkpoints (PD-1 and LAG-3) based on the expression profile data of The Cancer Genome Atlas. As shown in online supplemental figure S8A,B, the expression of TIGIT was positively correlated with the expression of PD-1 or LAG-3 in pan-cancers (33 types) and some of them, including but not limited to breast cancer, liver hepatocellular carcinoma, and colorectal carcinoma. Next, by treating tumor cells with VV-control or VV-scFv-TIGIT, we also investigated whether the infection of these two VVs affected 
A

Intraperitoneal inoculation of $\mathrm{H} 22$ cells $\left(1 \times 10^{7}\right.$ cells/mouse $)$

Intraperitoneal injection of CD8- or NK1.1-mAB (500ug/mouse)

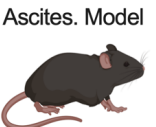

C57BL/6 mice

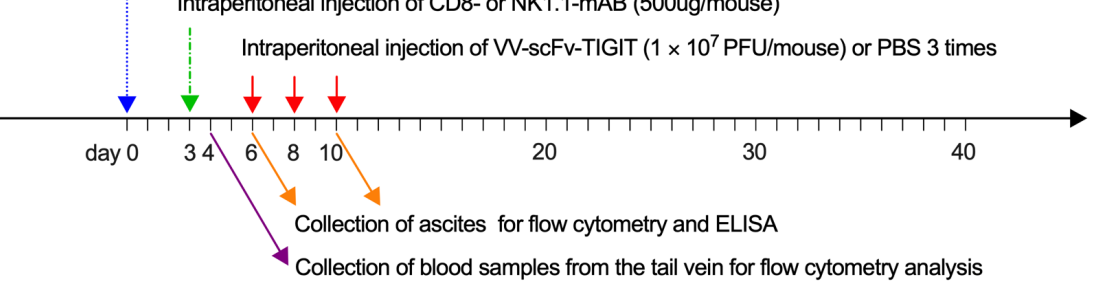

B

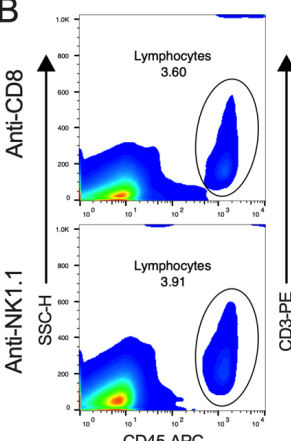

CD45-APC

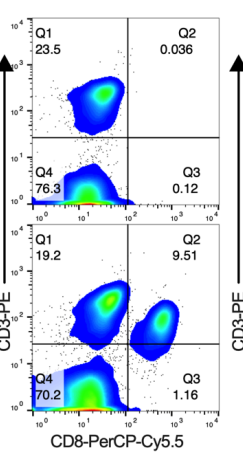

CD8-PerCP-Cy5.5

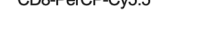

。
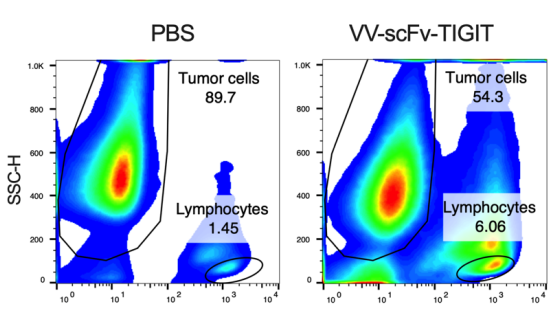

$E$
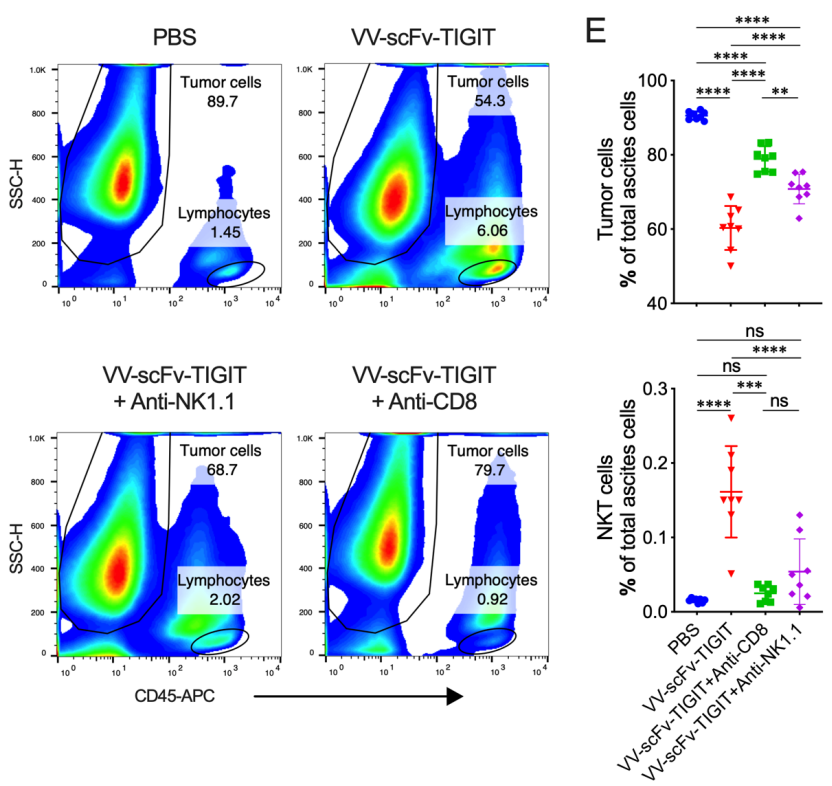

F
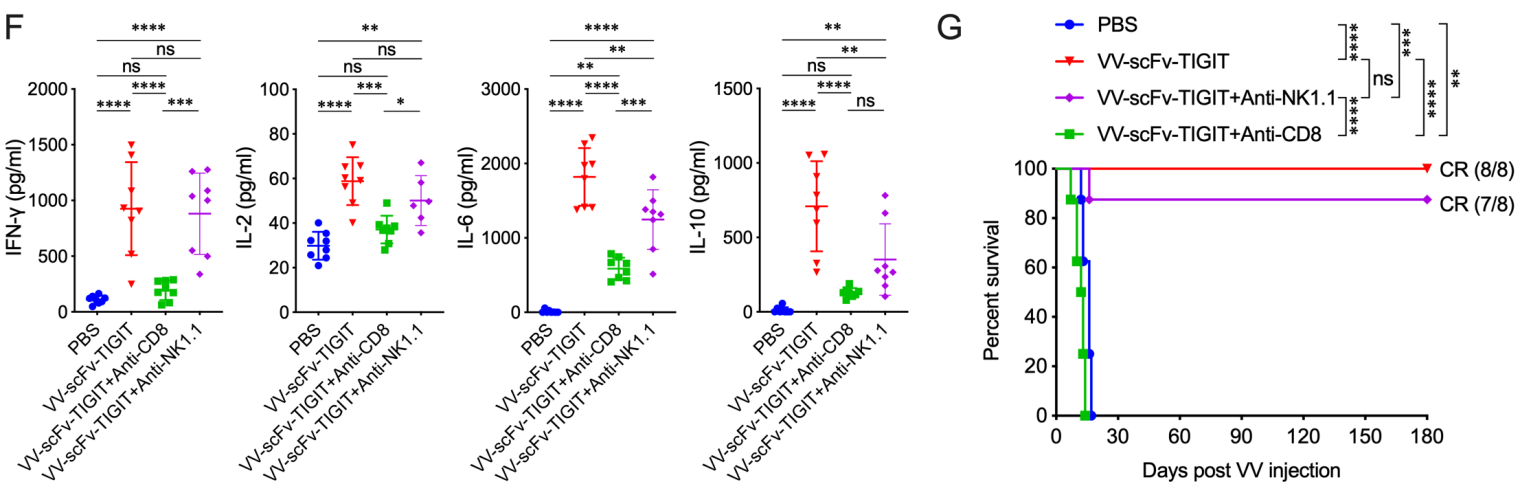

Figure $5 \mathrm{CD}^{+} \mathrm{T}$ cells mediated the antitumor immunity of VV-scFv-TIGIT. (A) Treatment scheme of H22 ascites model. (B, C) The depletion of CD8 ${ }^{+}$T cells and NK cells in the blood (B) or ascites (C) of mice was analyzed by flow cytometry. (D) Representative diagram of flow cytometric analysis of tumor cells and lymphocytes in the ascites. (E) Flow cytometric analysis of the proportion of tumor cells, lymphocytes, and their subpopulations in the ascites. (F) The levels of cytokines in the ascites were detected by ELISA. (G) Kaplan-Meier survival curves of tumor-bearing mice. ns, no significant differences; ${ }^{*} \mathrm{p}<0.05 ;{ }^{* \star} \mathrm{p}<0.01 ;{ }^{* \star \star} \mathrm{p}<0.001 ;{ }^{* \star \star \star} \mathrm{p}<0.0001$. PBS, phosphate-buffered saline; scFv, single-chain variable fragment; TIGIT, T-cell immunoglobulin and ITIM domain; VV, vaccinia virus. 
A

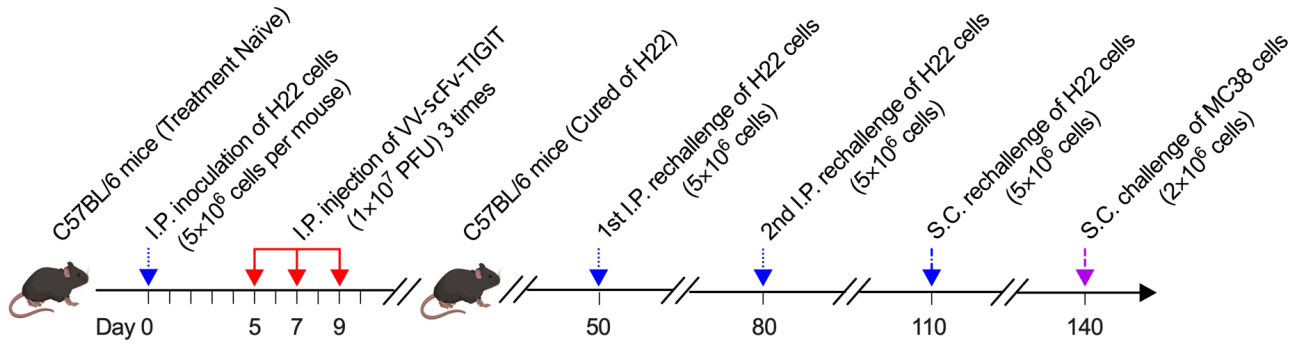

B

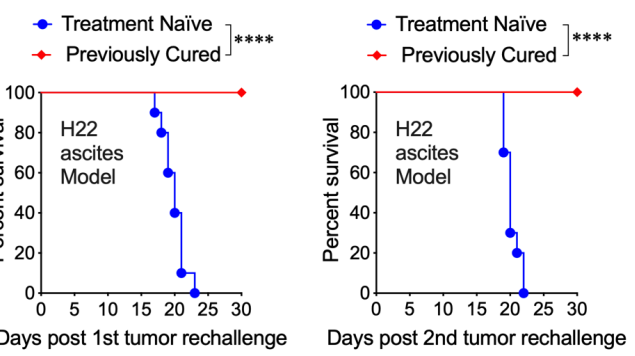

C
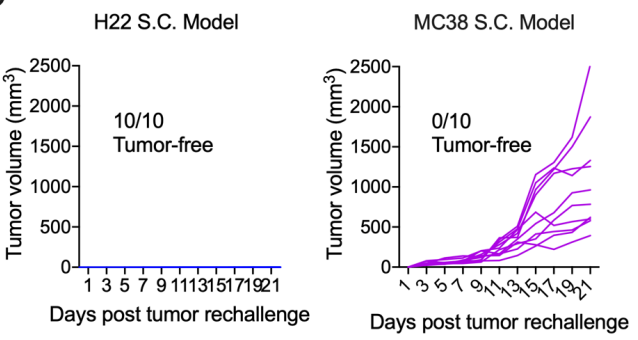

D
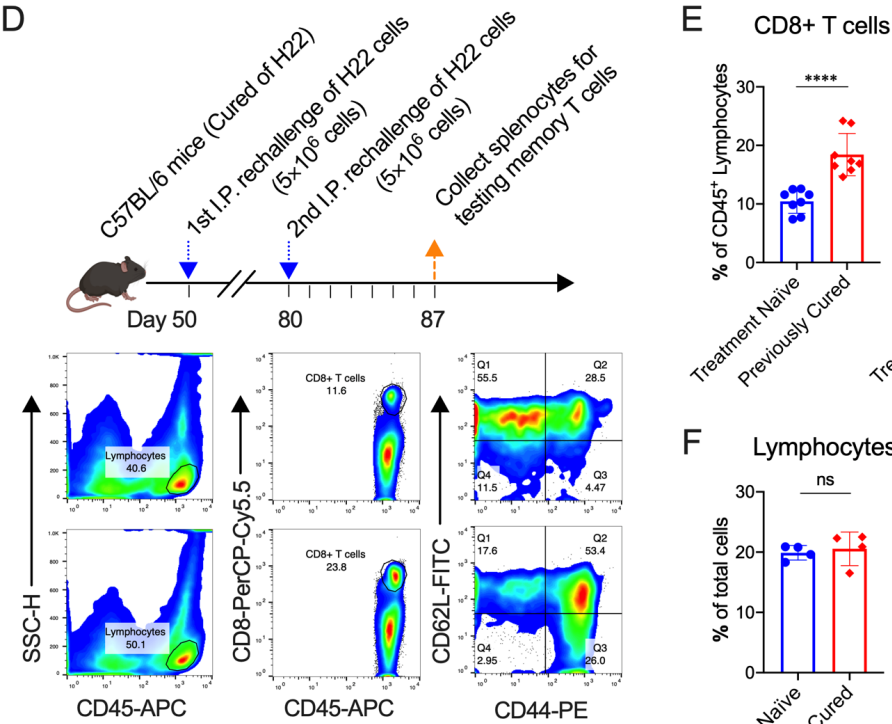

Tnaïve
$\left(\right.$ CD62L $\left.^{+} \mathrm{CD} 44^{-}\right)$

Tcm
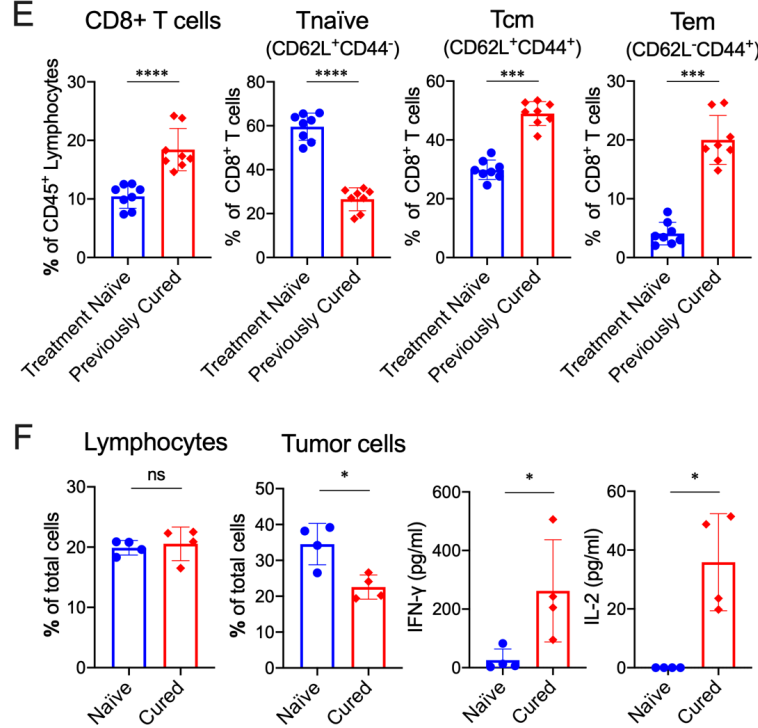

Figure 6 Treatment of mice with VV-scFv-TIGIT established long-term tumor-specific immunological memory. (A) Rechallenge scheme. (B) Kaplan-Meier survival curves of the treatment-naïve mice or the mice cured of H22 by VV-scFv-TIGIT. (C) Tumor volumes of previously cured mice rechallenged with $\mathrm{H} 22$ or MC38 cells subcutaneously. (D) Rechallenge scheme and representative diagram of flow cytometric analysis of memory T cells. (E) Flow cytometric analysis of the proportion of CD8 ${ }^{+} \mathrm{T}$ cells, naïve $\left(\mathrm{CD} 2 \mathrm{~L}^{+} \mathrm{CD} 44^{-}\right)$, effector memory $\left(\mathrm{CD} 62 \mathrm{~L}^{-} \mathrm{CD} 44^{+}\right)$and central memory $\left(\mathrm{CD} 62 \mathrm{~L}^{+} \mathrm{CD} 44^{+}\right) \mathrm{CD}^{+} \mathrm{T}$ cells. (F) Co-culture of $\mathrm{H} 22$ cells with splenocytes from the H22 rechallenged mice. The proportion of lymphocytes and tumor cells in the co-culture system was detected by flow cytometry. The levels of cytokines in the co-culture system were detected by ELISA. ns, no significant differences; ${ }^{*} \mathrm{p}<0.05 ;{ }^{* \star *} \mathrm{p}<0.001 ;{ }^{* \star \star *} \mathrm{p}<0.0001$. scFv, single-chain variable fragment; TIGIT, T-cell immunoglobulin and ITIM domain; VV, vaccinia virus.

the expression of PD-L1 on 4T1, CT26, MC38, and H22 cells (online supplemental figure S9). After 48 hours of treatment, the two viruses showed similar infection efficiency in the four tumor cell lines, in which $>70 \%$ of $4 \mathrm{~T} 1$ cells were infected by the VVs and expressed EGFP, while $50 \%-60 \%$ of CT26 or MC38 cells were infected, and only $20 \%$ of H22 were infected. In response to VV infection, the expression of PD-L1 on the surface of the four tumor cell lines was upregulated and showed a similar degree of upregulation between the two viruses.

To explore whether the combined application of $\alpha$ PD- 1 could enhance the antitumor efficacy of VV-scFv-TIGIT, we used an intratumoral injection of VV-scFv-TIGIT and intraperitoneal injection of $\alpha \mathrm{PD}-1$ to treat the MC38 subcutaneous tumor model (online supplemental figure $\mathrm{S} 10 \mathrm{~A})$. As shown in online supplemental figure S10B, monotherapy with VV-scFv-TIGIT or $\alpha \mathrm{PD}-1$ significantly inhibited tumor growth $(\mathrm{p}<0.01$ and $\mathrm{p}<0.01 \mathrm{vs} \mathrm{PBS})$. At the same time, there was no significant difference in the tumor volume of mice between the two monotherapies $(p>0.05)$. However, combined therapy with $\mathrm{VV}-\mathrm{scFv}$ TIGIT and $\alpha \mathrm{PD}-1$ further reduced the tumor volume of mice $(p<0.05 v$ v $\quad$ VV-scFv-TIGIT; $p<0.01$ vs $\alpha P D-1$; $p<0.001$ vs PBS), in which one mouse achieved tumor CR (online supplemental figure S10C). Consistent with these results, monotherapy with VV-scFv-TIGIT or $\alpha$ PD-1 and 
the combination therapy with both of them significantly prolonged the survival time of mice $(\mathrm{p}<0.05, \mathrm{p}<0.05$, and $\mathrm{p}<0.01$ vs $\mathrm{PBS}$ ). However, there was no significant difference in survival among these three groups of mice (online supplemental figure S10D).

We also evaluated the antitumor activity of the combined application of VV-scFv-TIGIT and $\alpha$ PD- 1 on the CT26 colon cancer model (figure 7A). Monotherapy with $\alpha \mathrm{PD}-1$ or VV-scFv-TIGIT and the combination of the two significantly inhibited tumor growth $(\mathrm{p}<0.05, \mathrm{p}<0.05$ or $\mathrm{p}<0.01 \mathrm{vs}$ PBS). The combination therapy showed a trend of smaller tumor volume than mice treated with VV-scFv-TIGIT or $\alpha$ PD-1 alone (figure 7B). One-ninth of $\alpha \mathrm{PD}$-1-treated, two-ninths of VV-scFv-TIGIT-treated, and five-ninths of co-treated mice achieved tumor CR, and one-ninth of the co-treated mice achieved partial remission (PR), while none of the eight mice treated with PBS achieved CR or PR (figure 7C). Consistent with these results, monotherapy with VV-scFv-TIGIT or $\alpha$ PD-1 significantly prolonged the survival time of the tumor-bearing mice ( $<<0.05$ or $p<0.01$ vs PBS). Moreover, the combination therapy further prolonged the survival time of the tumor-bearing mice $(p<0.05$ vs VV-scFv-TIGIT; $p<0.05$ vs $\alpha \mathrm{PD}-1 ; \mathrm{p}<0.001$ vs PBS; figure 7D).

Next, we evaluated the antitumor efficacy of the combined application of VV-scFv-TIGIT and $\alpha$ LAG-3 on the CT26 model (figure 7E). Consistent with the previous results, intratumoral injection of VV-control or VV-scFvTIGIT significantly inhibited tumor growth and prolonged the survival time of the tumor-bearing mice, whereas monotherapy with $\alpha \mathrm{LAG}-3$ did not show a stronger antitumor efficacy than PBS (figure 7F). Seven of 10 mice treated with VV-scFv-TIGIT plus $\alpha$ LAG-3 achieved tumor CR, whereas only 1 of 10 mice treated with $\alpha$ LAG-3 and 3 of 10 mice treated with VV-scFv-TIGIT achieved tumor CR (figure 7G). Consistent with these results, the combination therapy with $\alpha$ LAG-3 and VV-scFv-TIGIT significantly prolonged the survival time of mice compared with monotherapy with $\alpha \mathrm{LAG}-3$ or VV-scFv-TIGIT. Moreover, mice treated with VV-scFv-TIGIT plus $\alpha$ LAG-3 showed a longer survival time than mice treated with VV-control plus $\alpha$ LAG-3 (figure $7 \mathrm{H}$ ). These results indicated that the combined treatment with $\alpha \mathrm{PD}-1$ or $\alpha \mathrm{LAG}-3$ further improved the antitumor efficacy of VV-scFv-TIGIT.

\section{DISCUSSION}

The most prominent ability of OVs is that they can turn 'cold' tumors into 'hot' tumors, thereby providing a prerequisite immune microenvironment for immune checkpoint targeted therapy. ${ }^{34}$ Therefore, the combination therapy using OVs and ICBs has become a new and promising cancer immunotherapy strategy. ${ }^{35}$ One combination approach of this strategy is the intratumoral injection of OV plus systemic administration of ICBs. ${ }^{15}$ Another combination approach is to modify the OV with the gene that encodes the immune checkpoint blockade so that the OV infects tumor cells and locally releases the immune checkpoint blockade. ${ }^{20}$ Both of these combination approaches have been shown to cause a synergistic effect of the OV and ICBs in the treatment of cancer. ${ }^{15} 2036$ Previous studies have shown that the combined use of local injection of oncolytic VV and systemic administration of PD-1 blockade has higher efficacy than either monotherapy. ${ }^{737}$ Alternatively, engineered oncolytic VV or HSV expressing the PD-1 blockades also showed the equal antitumor effect as the combined use of $\mathrm{OV}$ and PD-1 blockades. ${ }^{19}{ }^{23}$ In this study, we demonstrated for the first time that armed $\mathrm{VV}$ with a scFv against TIGIT significantly enhanced the antitumor efficacy of the parental VV by remodeling the immune status of the TME. We also demonstrated for the first time that the additional combination of PD-1 or LAG-3 blockade further enhanced the antitumor efficacy of $\mathrm{VV}$ armed with the $\mathrm{scFv}$ against TIGIT.

The previous study has shown that intratumoral injection of the control VV with TK deletion but not expressing any foreign genes resulted in the remodeling of the tumor immune microenvironment, particularly with the increasing of T cells. ${ }^{38}$ In the present study, we found that the control VV has only limited ability to recruit TILs in both subcutaneous and ascites tumor models. A recent study also obtained similar results in CT26, B16F10, and LLC subcutaneous tumor models, even with virus titers twice as high as ours. ${ }^{39}$ These findings emphasized the need to use additional immunomodulators to genetically modify VV to further improve its lymphocyte recruitment function. The most extensively used transgene is the cytokine GM-CSF, which has been shown to be effective in increasing the infiltration of T-cells and macrophages. ${ }^{7}$ Besides, it has been shown that the vectorization of IL cytokines (such as IL-2, IL-12, or IL-15) in VV significantly enhanced its ability to recruit $\mathrm{T}$ cells and NK cells. ${ }^{40-42}$ To our surprise, arming VV with scFv-TIGIT significantly improved its ability to recruit TILs in both subcutaneous and ascites tumor models. In theory, scFv-TIGIT should not have the function of directly recruiting $\mathrm{T}$ cells. We suspect that this may be due to scFv-TIGIT relieving the immunosuppressive status of the $\mathrm{CD}^{+} \mathrm{T}$ cells, thereby enhancing the secretion of inflammatory cytokines and chemokines and allowing other lymphocytes to recruit into the tumor.

Compared with the subcutaneous tumor model, the ascites tumor model is a more excellent model, which can dynamically monitor the oncolytic effect of the virus and the immune response triggered by the virus by repeatedly obtaining ascites without sacrificing mice. ${ }^{43}$ Through dynamic observation, we found that the cell composition in the ascites of the VV-control-treated mice was still dominated by tumor cells, although it also showed a gradual increase in lymphocytes and a gradual decrease in tumor cells. However, treatment with VV-scFv-TIGIT eventually led to the disappearance of tumor cells in the ascites of mice, accompanied by a high degree of $\mathrm{CD} 45^{+}$immunecell infiltration, indicating that the infiltration and activation of immune cells, especially $\mathrm{CD} 8^{+} \mathrm{T}$ cells, is a more 
A

Subcutaneous inoculation of CT26 cells $\left(5 \times 10^{5}\right.$ cells/mouse)

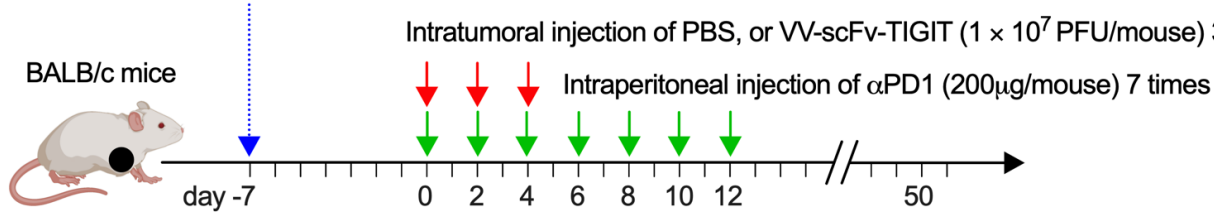

B

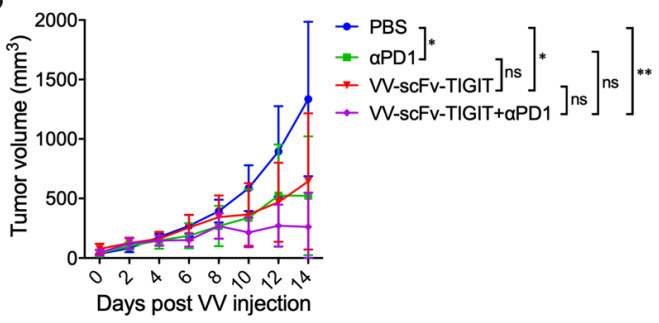

C

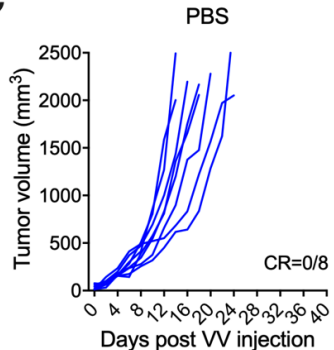

$\mathrm{D}$

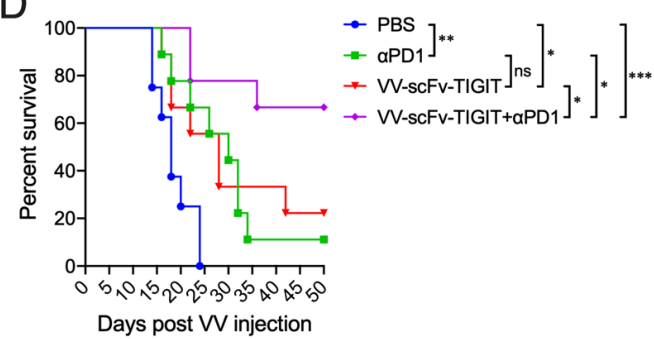

PD1

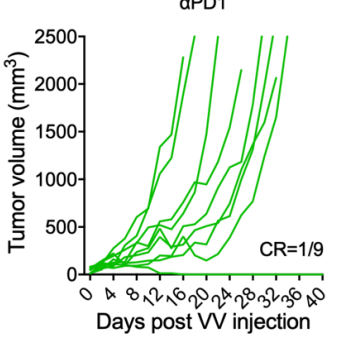

W-scFv-TIGIT

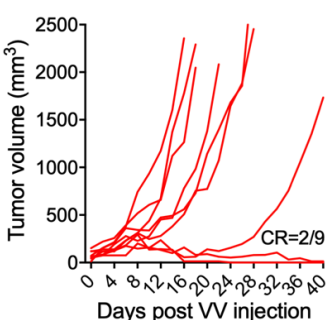

W-scFv-TIGIT+ aPD1

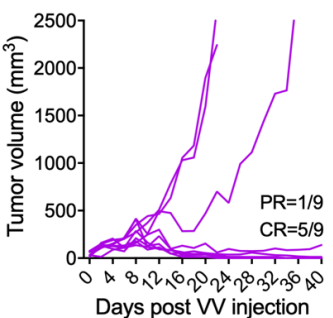

E

Subcutaneous inoculation of CT26 cells $\left(5 \times 10^{5}\right.$ cells/mouse $)$

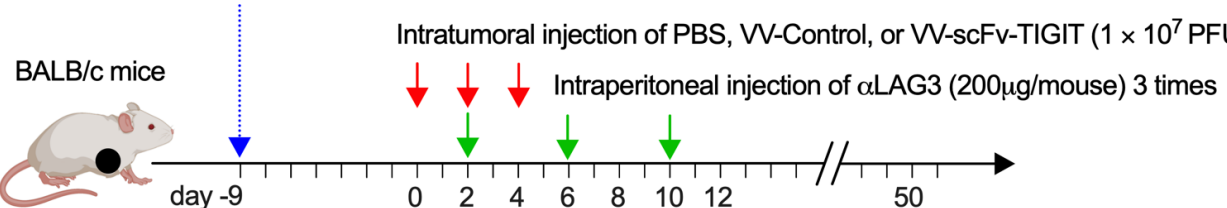

$\mathrm{F}$

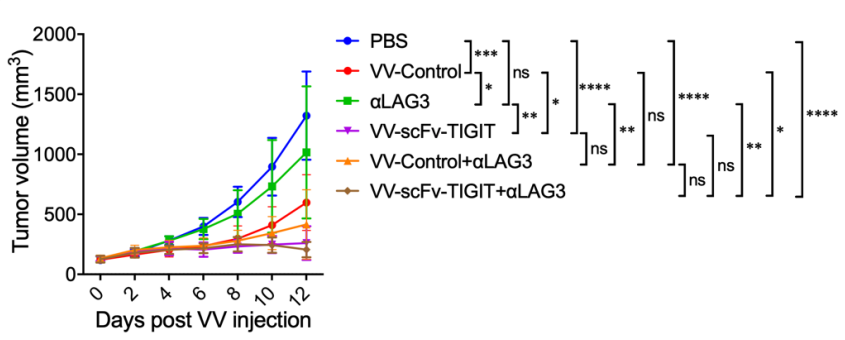

G

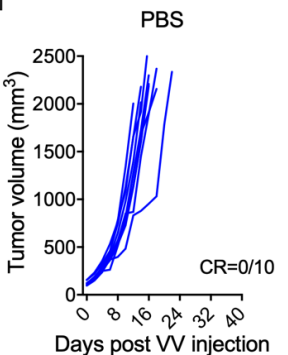

W-Control

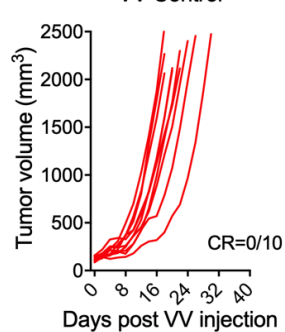

$\mathrm{H}$

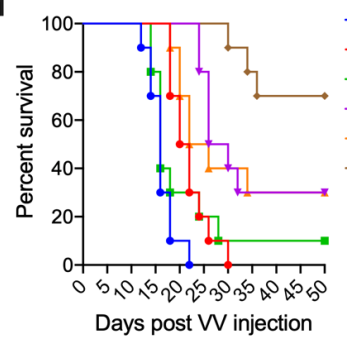

Figure 7 Combination therapy with $\alpha \mathrm{PD}-1$ or $\alpha$ LAG-3 improved the antitumor efficacy of VV-scFv-TIGIT in colon cancer models (A) Treatment scheme of CT26 subcutaneous (S.C.) tumor model with VV and $\alpha$ PD-1. (B) Mean tumor volume of mice. (C) Individual tumor growth curve of mice. (D) Kaplan-Meier survival curves of tumor-bearing mice. (E) Treatment scheme of CT26 S.C. tumor model with VV and $\alpha$ LAG-3. (F) Mean tumor volume of mice. (G) Individual tumor growth curve of mice. (H) Kaplan-Meier survival curves of tumor-bearing mice. Tumor volume was measured every 2 days. Error bars represent SD. Once the tumor volume exceeded $2000 \mathrm{~mm}^{3}$, the mouse was considered dead. CR, complete remission; PR, partial remission; ns, no significant differences; ${ }^{*} \mathrm{p}<0.05 ;{ }^{* \star} \mathrm{p}<0.01 ;{ }^{* \star *} \mathrm{p}<0.001 ;{ }^{* \star \star *} \mathrm{p}<0.0001$. PBS, phosphate-buffered saline; scFv, single-chain variable fragment; TIGIT, T-cell immunoglobulin and ITIM domain; VV, vaccinia virus. 
important antitumor mechanism relative to the direct oncolytic effect of the virus. ${ }^{43-45}$ Consistent with these findings, we found that treatment of mouse ascites tumor with VV-scFv-TIGIT led to a higher degree of activation of the tumor-infiltrating $\mathrm{CD}^{+} \mathrm{T}$ cells with expressing higher levels of CD107A, IFN- $\gamma$, TNF- $\alpha$, and granzyme B. Furthermore, the depletion of $\mathrm{CD}^{+} \mathrm{T}$ cells eliminated the therapeutic efficacy of VV-scFv-TIGIT, which was accompanied by a significant reduction of the percentage of $\mathrm{CD}^{+} \mathrm{T}$, $\mathrm{CD}^{+} \mathrm{T}, \mathrm{NK}$, and NKT cells and a significant reduction of secretion of IFN- $\gamma$, IL- 6 , and IL-10 in the ascites. Our study confirmed that the activation of $\mathrm{CD}^{+} \mathrm{T}$ cells has an indispensable role in anticancer immunity induced by $\mathrm{VV}^{739}$

In a previous study, the control VV significantly increased the proportion of NK cells in both B16F10 and CT26 tumors. ${ }^{39}$ However, in the present study, we did not find that the control VV and VV-scFv-TIGIT increased the proportion of NK cells in the CT26 model. We suspect that the reason for the difference may be the different doses of VV because we only used a quarter of it. Another previous study has shown that early depletion (before tumor implantation) rather than late depletion of NK cells abolished the efficacy of Newcastle disease virus plus anti-PD-1 therapy on B16-F10 tumor mice, indicating that NK cells are very important in the early stages of the OV treatment process. ${ }^{46}$ In our study, the depletion of NK cells did not significantly reduce the therapeutic efficacy of VV-scFv-TIGIT, which indicates that NK cells are not the main lymphocyte subset that mediates the antitumor immunity of VV-scFv-TIGIT. These results are consistent with our previously published results. Although oncolytic $\mathrm{ADV}$ increased the proportion of NK cells in the mouse liver cancer model, depletion of NK cells did not significantly affect the antitumor effect of the oncolytic ADV encoding CD137-PDL1 soluble fusion protein. ${ }^{43}$ Because TIGIT has been proven to be a checkpoint receptor for NK cells, and blocking the 'PVR-TIGIT axis' can prevent NK cell exhaustion and elicit potent antitumor immunity ${ }^{4748}$ their role should not be ignored in future clinical trials.

In this study, we found that although VV-scFv-TIGIT inhibited the growth of H22 subcutaneous tumors, it did not make the tumor regress completely. However, whether in $\mathrm{BALB} / \mathrm{c}$ or $\mathrm{C} 57 \mathrm{BL} / 6$ mice, VV-scFv-TIGIT induced a complete response to ascites tumors, which seems to indicate that ascites tumors are easier to be cured than subcutaneous tumors. Similar to our research, chimeric antigen receptor-modified $\mathrm{T}$ (CAR-T) cells have been reported to have good efficacy on hematological tumors, but have poor efficacy on solid tumors. ${ }^{49}$ In solid tumors, CAR-T cells must overcome multiple obstacles to reach the tumor site. However, in hematological tumors, the infused CAR T can directly recognize and kill tumor cells in the blood. ${ }^{50}$ In the same way, the liquid environment of the ascites tumor might facilitate the recognition of tumor cells by immune cells. Therefore, we hypothesized that indirect treatment of primary tumors by treating the ascites they formed may be more effective. To confirm this hypothesis, we established an ascites-subcutaneous dual tumor model and then used an intraperitoneal injection of the OV for treatment. As expected, treatment of the ascites resulted in a complete response of the subcutaneous tumor. Consistent with these results, we also found that mice with ascites cured by OV formed immunological memory and rejected subcutaneous re-inoculation with the same tumor cells. This 'attack-across-the-mountain' strategy may be more suitable for certain cancers that prefer to form ascites, such as ovarian, liver, and colon cancers, especially for individuals whose primary tumors are difficult to be surgically removed.

Studies have shown that activation of $\mathrm{T}$ cells is always accompanied by the upregulation of immune checkpoint molecules such as PD-1, TIM3, LAG3, and TIGIT, which will inhibit the excessive activation of $\mathrm{T}$ cells and maintain the immune homeostasis. ${ }^{51}$ In this study, we found that VV-scFv-TIGIT upregulated all these immune checkpoint molecules in ascites tumors, and the upregulation of these molecules on $\mathrm{CD}^{+} \mathrm{T}$ cells did not affect the therapeutic efficacy of VV. Compared with PD-1, we observed a more significant increase in LAG-3 expression on tumorinfiltrating $\mathrm{CD}^{+} \mathrm{T}$ cells after $\mathrm{VV}$-scFv-TIGIT virotherapy in both ascites and subcutaneous tumors. In ascites tumors, although VV treatment resulted in the increase of LAG-3 on the surface of $\mathrm{CD}^{+} \mathrm{T}$ cells, it did not affect $\mathrm{CD}^{+} \mathrm{T}$ cells expressing high levels of IFN- $\gamma$, TNF- $\alpha$, granzyme B, and CD107A. Our findings are consistent with previous work that vaccination with $\mathrm{VV}$ increases the intracellular expression of LAG-3 in CD8 T cells. ${ }^{52}$ Furthermore, they highlighted that $\mathrm{CD}^{+} \mathrm{T}$ cells expressed high levels of LAG-3 are still functional and capable of producing effector cytokines. ${ }^{53}$ The liquid environment of ascites tumors may be conducive to these cytokines, making them less affected by the negative immune regulation of LAG-3. Unlike hematological or ascites tumors, solid tumors often have a more complex immunosuppressive TME, ${ }^{54}$ the negative immunomodulatory function of LAG-3 may play a greater role by affecting the proliferation of $\mathrm{CD} 8^{+}$ T cells. ${ }^{52}$ In our study, the additional combined use of LAG-3 antibody significantly enhanced the therapeutic efficacy of VV-scFv-TIGIT on the CT26 model, suggesting that dual scFv-armed VV targeting TIGIT and LAG-3 may be a better virus engineering strategy.

In this study, we found that infection with VV upregulated the expression of PD-L1 on the surface of tumor cells. Although PD-L1 usually downregulates the activity of $\mathrm{T}$ cells, ${ }^{5556}$ studies have shown that patients with higher PD-1/PD-L1 basic expression on tumors are more responsive to anti-PD-1/PD-L1 treatment. ${ }^{5758}$ In a recent study, $70 \%$ of MC38 subcutaneous tumors completely responded to an oncolytic VV co-expressing GM-CSF and PD-L1 inhibitor (a fusion protein of PD-1 extracellular domain and the Fc fragment) in combination with a PD-1 blockade. ${ }^{20}$ In another study, treatment with oncolytic VV co-expressing IL-7 and IL-12 in combination with PD-1 blockade resulted in complete remission of $100 \%$ 
of CT26 subcutaneous tumors. ${ }^{39}$ In the present study, although the additional combination of PD-1 blockade significantly improved the therapeutic effect of $\mathrm{VV}-\mathrm{scFv}$ TIGIT on MC38 and CT26 subcutaneous tumors, the complete response rate was lower than the previous two studies. One possible reason is that the dose of $\mathrm{VV}$ we used in this study was lower than that used in the two previous studies (approximately one-half). More importantly, the two previous studies have armed the VV with cytokines, ${ }^{20}{ }^{39}$ which can perform biological functions at very low concentrations (pg level), and in most cases, cascade amplification effects will occur. However, our recombinant VV has only inserted immune checkpoints, which may be an important defect in our study. Moreover, due to technical limitations, we did not obtain a dual scFv armed VV. In the future, further genetic engineering is needed to improve the therapeutic efficacy of our virus, such as co-expression of ICBs and cytokines related to the immune-cell activation or exploring arming the VV with two immune checkpoints.

In conclusion, our data indicated that oncolytic virotherapy using engineered VV-scFv-TIGIT was an effective strategy for cancer immunotherapy. Intratumoral injection of $\mathrm{VV}$-scFv-TIGIT caused a profound reshaping of the TME from 'cold' to 'hot' status. VV-scFv-TIGIT also synergized with PD-1 or LAG-3 blockade to achieve a complete response to tumors with poor response to $\mathrm{VV}$ or ICB monotherapy.

Acknowledgements The authors would like to thank Dr Xiaofei Li (Nanjing Medical University) for conducting the ELISA assay.

Contributors SZ and JiwuW designed the study. SZ, MW, TX, LK, BH, ShiqunW, and ShibingW designed and performed the experiments. SZ, MW, TX, and BH analyzed the data. SZ wrote the manuscript. JD, JunhuaW, and JiwuW contributed to study supervision. SZ and JiwuW critically review the manuscript. All authors read and approved the final manuscript. JiwuW accepts full responsibility for the work and/ or the conduct of the study, had access to the data, and controlled the decision to publish.

Funding This study was supported by the National Natural Science Foundation of China (81773255, 81472820, and 81700037), Science and Technology Innovation Foundation of Nanjing University (14913414), Natural Science Foundation of Jiangsu Province of China (BK20171098), and Nanjing University Innovation Program for the PhD Candidate, and Research Foundation for Advanced Talents of Chifeng Municipal Hospital.

Competing interests None declared.

Patient consent for publication Not applicable.

Ethics approval All animal procedures and experiments were performed following the guidelines that had been approved by the Animal Care and Use Committee of the Medical School of Nanjing University.

Provenance and peer review Not commissioned; externally peer reviewed.

Data availability statement Data sharing not applicable as no datasets generated and/or analyzed for this study.

Supplemental material This content has been supplied by the author(s). It has not been vetted by BMJ Publishing Group Limited (BMJ) and may not have been peer-reviewed. Any opinions or recommendations discussed are solely those of the author(s) and are not endorsed by BMJ. BMJ disclaims all liability and responsibility arising from any reliance placed on the content. Where the content includes any translated material, BMJ does not warrant the accuracy and reliability of the translations (including but not limited to local regulations, clinical guidelines, terminology, drug names and drug dosages), and is not responsible for any error and/or omissions arising from translation and adaptation or otherwise.
Open access This is an open access article distributed in accordance with the Creative Commons Attribution Non Commercial (CC BY-NC 4.0) license, which permits others to distribute, remix, adapt, build upon this work non-commercially, and license their derivative works on different terms, provided the original work is properly cited, appropriate credit is given, any changes made indicated, and the use is non-commercial. See http://creativecommons.org/licenses/by-nc/4.0/.

ORCID iD

Shuguang Zuo http://orcid.org/0000-0001-9649-1200

\section{REFERENCES}

1 Chiocca EA, Rabkin SD. Oncolytic viruses and their application to cancer immunotherapy. Cancer Immunol Res 2014;2:295-300.

2 Kaufman HL, Kohlhapp FJ, Zloza A. Oncolytic viruses: a new class of immunotherapy drugs. Nature Reviews Drug Discovery 2015;14:642-62.

3 Guo ZS, Lu B, Guo Z, et al. Vaccinia virus-mediated cancer immunotherapy: cancer vaccines and oncolytics. J Immunother Cancer 2019;7:6.

4 Howells A, Marelli G, Lemoine NR, et al. Oncolytic viruses-interaction of virus and tumor cells in the battle to eliminate cancer. Front Oncol 2017;7:195.

5 Marelli G, Howells A, Lemoine NR, et al. Oncolytic viral therapy and the immune system: a double-edged sword against cancer. Front Immunol 2018;9:866.

6 Park B-H, Hwang T, Liu T-C, et al. Use of a targeted oncolytic poxvirus, JX-594, in patients with refractory primary or metastatic liver cancer: a phase I trial. Lancet Oncol 2008;9:533-42.

7 Chon $\mathrm{HJ}$, Lee WS, Yang $\mathrm{H}$, et al. Tumor microenvironment remodeling by intratumoral oncolytic vaccinia virus enhances the efficacy of Immune-Checkpoint blockade. Clin Cancer Res 2019;25:1612-23.

8 Kaufman HL, Kohlhapp FJ, Zloza A. Oncolytic viruses: a new class of immunotherapy drugs. Nat Rev Drug Discov 2015;14:642-62.

9 Raja J, Ludwig JM, Gettinger SN, et al. Oncolytic virus immunotherapy: future prospects for oncology. J Immunother Cancer 2018;6:140.

10 Andtbacka RHI, Kaufman HL, Collichio F, et al. Talimogene Laherparepvec improves durable response rate in patients with advanced melanoma. J Clin Oncol 2015;33:2780-8.

11 Zheng $M$, Huang J, Tong A, et al. Oncolytic viruses for cancer therapy: barriers and recent advances. Mol Ther Oncolytics 2019;15:234-47.

12 Qin S, Xu L, Yi M, et al. Novel immune checkpoint targets: moving beyond PD-1 and CTLA-4. Mol Cancer 2019;18:155.

13 Bommareddy PK, Shettigar M, Kaufman HL. Integrating oncolytic viruses in combination cancer immunotherapy. Nat Rev Immunol 2018;18:498-513.

14 Puzanov I, Milhem MM, Minor D, et al. Talimogene laherparepvec in combination with ipilimumab in previously untreated, unresectable stage IIIB-IV melanoma. J Clin Oncol 2016;34:2619-26.

15 Ribas A, Dummer R, Puzanov I, et al. Oncolytic virotherapy promotes intratumoral T cell infiltration and improves anti-PD-1 immunotherapy. Cell 2017;170:1109-19.

16 Malhotra S, Kim T, Zager J, et al. Use of an oncolytic virus secreting GM-CSF as combined oncolytic and immunotherapy for treatment of colorectal and hepatic adenocarcinomas. Surgery 2007;141:520-9.

17 Pearl TM, Markert JM, Cassady KA, et al. Oncolytic virus-based cytokine expression to improve immune activity in brain and solid tumors. Mol Ther Oncolytics 2019;13:14-21.

$18 \mathrm{Li} J, \mathrm{O}^{\prime}$ Malley M, Urban J, et al. Chemokine expression from oncolytic vaccinia virus enhances vaccine therapies of cancer. Mol Ther 2011;19:650-7.

19 Zhu Y, Hu X, Feng L, et al. Enhanced therapeutic efficacy of a novel oncolytic herpes simplex virus type 2 encoding an antibody against programmed cell death 1. Mol Ther Oncolytics 2019;15:201-13.

20 Wang G, Kang X, Chen KS, et al. An engineered oncolytic virus expressing PD-L1 inhibitors activates tumor neoantigen-specific $T$ cell responses. Nat Commun 2020;11:1395.

21 Bartee MY, Dunlap KM, Bartee E. Tumor-localized secretion of soluble PD1 enhances oncolytic virotherapy. Cancer Res 2017;77:2952-63.

22 Vijayakumar G, McCroskery S, Palese P. Engineering Newcastle disease virus as an oncolytic vector for intratumoral delivery of immune checkpoint inhibitors and immunocytokines. J Virol 2020;94 doi:10.1128/JVI.01677-19

23 Kleinpeter $\mathrm{P}$, Fend L, Thioudellet $\mathrm{C}$, et al. Vectorization in an oncolytic vaccinia virus of an antibody, a Fab and a scFv against 
programmed cell death -1 (PD-1) allows their intratumoral delivery and an improved tumor-growth inhibition. Oncoimmunology 2016;5:e1220467.

24 Dias JD, Hemminki O, Diaconu I, et al. Targeted cancer immunotherapy with oncolytic adenovirus coding for a fully human monoclonal antibody specific for CTLA-4. Gene Ther 2012;19:988-98.

25 Jhawar SR, Thandoni A, Bommareddy PK, et al. Oncolytic virusesnatural and genetically engineered cancer immunotherapies. Front Oncol 2017;7:202

26 Harjunpää H, Guillerey C. TIGIT as an emerging immune checkpoint. Clin Exp Immunol 2020;200:108-19.

27 Sanchez-Correa B, Valhondo I, Hassouneh F, et al. Dnam-1 and the TIGIT/PVRIG/TACTILE axis: novel immune checkpoints for natural killer cell-based cancer immunotherapy. Cancers 2019;11 doi:10.3390/cancers11060877

28 Chan CJ, Andrews DM, Smyth MJ. Receptors that interact with nectin and nectin-like proteins in the immunosurveillance and immunotherapy of cancer. Curr Opin Immunol 2012;24:246-51.

29 Chauvin J-M, Pagliano O, Fourcade J, et al. TIGIT and PD-1 impair tumor antigen-specific CD8 ${ }^{+} \mathrm{T}$ cells in melanoma patients. J Clin Invest 2015;125:2046-58.

30 Kurtulus S, Sakuishi K, Ngiow S-F, et al. TIGIT predominantly regulates the immune response via regulatory T cells. J Clin Invest 2015;125:4053-62.

31 Rodriguez-Abreu D, Johnson ML, Hussein MA, et al. Primary analysis of a randomized, double-blind, phase II study of the anti-TIGIT antibody tiragolumab (tira) plus atezolizumab (atezo) versus placebo plus atezo as first-line (1L) treatment in patients with PD-L1-selected NSCLC (CITYSCAPE). J Clin Oncol 2020;38:9503. doi:10.1200/ JCO.2020.38.15_suppl.9503

32 Solomon BL, Garrido-Laguna I. TIGIT: a novel immunotherapy target moving from bench to bedside. Cancer Immunol Immunother 2018;67:1659-67.

33 Lin C, Ren W, Luo Y, et al. Intratumoral delivery of a PD-1-blocking scFv encoded in oncolytic HSV-1 promotes antitumor immunity and synergizes with TIGIT blockade. Cancer Immunol Res 2020;8:632-47.

34 Gujar S, Pol JG, Kroemer G. Heating it up: oncolytic viruses make tumors 'hot' and suitable for checkpoint blockade immunotherapies. Oncoimmunology 2018;7:e1442169.

35 Chiu M, Armstrong EJL, Jennings V, et al. Combination therapy with oncolytic viruses and immune checkpoint inhibitors. Expert Opin Biol Ther 2020;20:635-52.

36 Martin NT, Bell JC. Oncolytic virus combination therapy: killing one bird with two stones. Mol Ther 2018;26:1414-22.

37 Liu Z, Ravindranathan R, Kalinski P, et al. Rational combination of oncolytic vaccinia virus and PD-L1 blockade works synergistically to enhance therapeutic efficacy. Nat Commun 2017;8:14754.

38 Rivadeneira DB, DePeaux K, Wang Y, et al. Oncolytic viruses engineered to enforce leptin expression reprogram tumor-infiltrating $\mathrm{T}$ cell metabolism and promote tumor clearance. Immunity 2019;51:e544:548-60.

39 Nakao S, Arai Y, Tasaki M, et al. Intratumoral expression of IL-7 and IL-12 using an oncolytic virus increases systemic sensitivity to immune checkpoint blockade. Sci Trans/ Med 2020;12 doi:10.1126/ scitransImed.aax7992
40 Kowalsky SJ, Liu Z, Feist M, et al. Superagonist IL-15-armed oncolytic virus elicits potent antitumor immunity and therapy that are enhanced with PD-1 blockade. Mol Ther 2018;26:2476-86.

41 Liu Z, Ge Y, Wang H, et al. Modifying the cancer-immune set point using vaccinia virus expressing re-designed interleukin-2. Nat Commun 2018:9:4682.

42 Ge Y, Wang H, Ren J, et al. Oncolytic vaccinia virus delivering tethered IL-12 enhances antitumor effects with improved safety. $\mathrm{J}$ Immunother Cancer 2020;8.

43 Zhang $\mathrm{Y}$, Zhang $\mathrm{H}$, Wei $\mathrm{M}$, et al. Recombinant adenovirus expressing a soluble fusion protein PD-1/CD137L subverts the suppression of CD8 $^{+}$T cells in HCC. Mol Ther 2019;27:1906-18.

44 Bai Y, Hui P, Du X, et al. Updates to the antitumor mechanism of oncolytic virus. Thorac Cancer 2019;10:1031-5.

45 Lemos de Matos A, Franco LS, McFadden G. Oncolytic viruses and the immune system: the dynamic duo. Mol Ther Methods Clin Dev 2020;17:349-58.

46 Zamarin D, Ricca JM, Sadekova S, et al. PD-L1 in tumor microenvironment mediates resistance to oncolytic immunotherapy. $J$ Clin Invest 2018;128:1413-28.

47 Zhang Q, Bi J, Zheng X, et al. Blockade of the checkpoint receptor TIGIT prevents NK cell exhaustion and elicits potent anti-tumor immunity. Nat Immunol 2018;19:723-32.

48 Chauvin J-M, Ka M, Pagliano O, et al. IL15 stimulation with TIGIT blockade reverses CD155-mediated NK-cell dysfunction in melanoma. Clin Cancer Res 2020;26:5520-33.

49 Martinez M, Moon EK. CAR T cells for solid tumors: new strategies for finding, infiltrating, and surviving in the tumor microenvironment. Front Immunol 2019;10:128.

50 Marofi F, Motavalli R, Safonov VA, et al. CAR T cells in solid tumors: challenges and opportunities. Stem Cell Res Ther 2021;12:81.

51 Anderson AC, Joller N, Kuchroo VK. Lag-3, Tim-3, and TIGIT: coinhibitory receptors with specialized functions in immune regulation. Immunity 2016;44:989-1004.

52 Grosso JF, Kelleher CC, Harris TJ, et al. LAG-3 regulates CD8+ T cell accumulation and effector function in murine self- and tumortolerance systems. J Clin Invest 2007;117:3383-92.

53 Grosso JF, Goldberg MV, Getnet D, et al. Functionally distinct LAG-3 and PD-1 subsets on activated and chronically stimulated CD8 T cells. J Immunol 2009;182:6659-69.

54 Zhang E, Gu J, Xu H. Prospects for chimeric antigen receptormodified T cell therapy for solid tumors. Mol Cancer 2018;17:7.

55 Freeman GJ, Long AJ, Iwai Y, et al. Engagement of the PD-1 immunoinhibitory receptor by a novel B7 family member leads to negative regulation of lymphocyte activation. J Exp Med 2000;192:1027-34

56 Dong H, Strome SE, Salomao DR, et al. Tumor-associated B7H1 promotes T-cell apoptosis: a potential mechanism of immune evasion. Nat Med 2002;8:793-800.

57 Tumeh PC, Harview CL, Yearley JH, et al. PD-1 blockade induces responses by inhibiting adaptive immune resistance. Nature 2014;515:568-71.

58 Topalian SL, Taube JM, Anders RA, et al. Mechanism-driven biomarkers to guide immune checkpoint blockade in cancer therapy. Nat Rev Cancer 2016;16:275-87. 